\title{
On the Movement of Vertex Fixed Points in the Simple GA
}

\author{
Alden $\mathrm{H}$. Wright \\ Computer Science \\ University of Montana \\ Missoula, MT 59812 \\ alden.wright@umontana.edu
}

\author{
Tomáš Gedeon \\ Mathematical Sciences \\ Montana State University \\ Bozeman, MT 59714 \\ gedeon@math.montana.edu
}

\author{
J. Neal Richter \\ Computer Science \\ Montana State University \\ Bozeman, MT 59714 \\ richter@cs.montana.edu
}

\begin{abstract}
The Vose dynamical system model of the simple genetic algorithm models the behavior of this algorithm for large population sizes and is the basis of the exact Markov chain model. Populations consisting of multiple copies of one individual correspond to vertices of the simplex. For zero mutation, these are fixed points of the dynamical system and absorbing states of the Markov chain. For proportional selection, the asymptotic stability of vertex fixed points is understood from previous work. We derive the eigenvalues of the differential at vertex fixed points of the dynamical system model for tournament selection. We show that as mutation increases from zero, hyperbolic asymptotically stable fixed points move into the simplex, and hyperbolic asymptotically unstable fixed points move outside of the simplex. We calculate the derivative of local path of the fixed point with respect to the mutation rate for proportional selection. Simulation analysis shows how fixed points bifurcate with larger changes in the mutation rate and changes in the crossover rate.
\end{abstract}

\section{Categories and Subject Descriptors}

I.2.8 [Artificial Intelligence]: Problem Solving, Control Methods, and Search - Heuristic Methods; G.1.m [Mathematics of Computing]: Miscelaneous-Evolutionary Computation and Genetic Algorithms

\section{General Terms}

Theory, Algorithms

\section{Keywords}

Genetic Algorithms, Theory, Crossover, Selection, Tournament Selection, Dynamical Systems, Fixed Points, Bifurcation

\section{INTRODUCTION}

Permission to make digital or hard copies of all or part of this work for personal or classroom use is granted without fee provided that copies are not made or distributed for profit or commercial advantage and that copies bear this notice and the full citation on the first page. To copy otherwise, to republish, to post on servers or to redistribute to lists, requires prior specific permission and/or a fee.

FOGA'11, January 5-9, 2010, Schwarzenberg, Austria.

Copyright 2010 ACM FOGA draft ver 0.5 - built Mon, 19 Jul 2010 21:17:59 $-0600 \ldots \$ 10.00$.
As the name suggests, the infinite population model (IPM) of the simple genetic algorithm (SGA) is a deterministic dynamical systems model that describes the behavior of the SGA as the population size goes to infinity. It is also the basis for the exact mathematical description of the SGA, namely the exact Markov chain model. The infinite population model and the exact Markov chain model are primarily due to Michael Vose, but also to collaborators Gunar Liepins, Alden Wright, A. E. Nix, and others. See [13], [14], [17], [15], [6].

Subsection 2.8 gives an example of the use of dynamical systems models to predict the behavior of a genetic algorithm.

The SGA is a generational genetic algorithm over bit strings of length $\ell$. As developed by Vose in [13], it includes any mask-based crossover, a very general model of mutation, and proportional, ranking, or a special form of tournament selection. This paper extends the model to standard tournament selection. (The models for ranking and tournament selection assume that no two individuals have the same fitness.)

Vertices of the simplex (defined below) are fixed points of this dynamical system when there is zero mutation. Using the results of a paper of Vose and Wright [16], of section 11.3 of Vose's book [13], and section 2.7 of this paper, the asymptotic stability of these fixed points can be calculated when the fitness function can be calculated (such as when there is a formula for the fitness function). As the crossover rate increases, the number of stable vertex fixed points increases (or remains constant).

When a vertex fixed point is asymptotically stable, this means that when the infinite population model is started sufficiently close to the fixed point, it will converge to the the fixed point. Intuitively, it means that the when the SGA with a sufficiently large population size is started close to the fixed point, it is highly likely to converge to the uniform population corresponding to the fixed point. (When mutation is zero, the SGA Markov chain is absorbing, so the SGA actually converges.) Thus, one would expect that the infinite population model with a sufficiently low mutation rate when started at or sufficiently near the fixed point would converge to a point close to the fixed point. This paper proves this result. Thus, the SGA with a low mutation when started near to the fixed point is likely to stay close to the fixed point for a long time.

When a vertex fixed point is asymptotically unstable, this means that the infinite population model can be started arbitrarily close to the fixed point and diverge away from the the fixed point. This paper shows that as positive mutation 
is introduced into the infinite population model in this case, the fixed point moves outside of the simplex. Thus, when the infinite population model with a low mutation rate is started at or sufficiently near the fixed point, it will diverge away from the fixed point. The behavior of the SGA in this case is discussed in subsection 4.4.

Populations are represented as vectors over the integers in half-open interval $[0, n)$ where these integers correspond to length $\ell$ bit strings through their binary representation and where $n=2^{\ell}$. A population vector $x$ has the properties $\sum_{i} x_{i}=1$ and $x_{i} \geq 0$ for all $i \in[0, n)$. The relative frequency of the bit string $i$ in the population is $x_{i}$. The space of all possible population vectors is the $(n-1)$-simplex $\Lambda$ in $\mathbb{R}^{n}$. Thus, $\Lambda=\left\{x \in \mathbb{R}^{n}: \sum_{i} x_{i}=1\right.$ and $\left.x_{i} \geq 0\right\}$. The vertices of the simplex are the unit vectors in $\mathbb{R}^{n}$; the $i^{\text {th }}$ unit vector $e_{i}$ corresponds to a uniform population consisting only of individuals whose string representation is the binary string representation of $i$.

Following [13], we use $\mathbf{1}$ as a notation for a column vector of all ones of length $\ell$, which corresponds to a string of all ones. In particular, if $i \in[0, n)$, then $\mathbf{1}^{T} i$ denotes the number of ones in the binary representation of $i$. We also use 1 as a notation for a column vector of all ones of length $n=2^{\ell}$. The meaning of the 1 symbol should be clear from the context.

The bitwise-AND of bit strings $i$ and $j$ is denoted by $i \otimes j$, and the bitwise-OR of $i$ and $j$ is denoted by $i \oplus j$. The onescomplement of $i$ is denoted by $\bar{i}$.

Population vectors in the simplex can be viewed either as populations of indeterminate size, or as sampling distributions for the next generation of the finite-population GA. (The formula for the exact Markov chain model is simply the application of the multinomial theorem to this sampling distribution.)

The infinite population model is a discrete-time dynamical system where time steps correspond to generations of the SGA. The model is described by a continuously differentiable $\left(C^{1}\right)$ map $\mathcal{G}: \Lambda \rightarrow \Lambda$ which will be defined below. If $x$ is the current population of the SGA, then the next generation population is obtained by sampling from $\mathcal{G}(x)$.

The IPM is deterministic with trajectory $x, \mathcal{G}(x), \mathcal{G}^{2}(x), \ldots$ Vose [13] shows that the expected next finite population is $\mathcal{G}(x)$, he also has theorems that show that IPM is the limiting behavior of the SGA as the population size goes to infinity.

The $\mathcal{G}$ map extends naturally to a neighborhood of the simplex in $\mathbb{R}^{n}$, and we will use $\mathcal{G}$ to denote this extended map.

\section{FIXED POINTS OF 9}

The results of subsection 2.3, and of subsection 2.7 as applied to tournament selection, are new, while the other subsections relate previously published results.

The map $\mathcal{G}$ is the composition of a selection map $\mathcal{F}$ and a mixing map $\mathcal{M}$.

$$
\mathcal{G}=\mathcal{M} \circ \mathcal{F}
$$

In this section, we describe the mixing map $\mathcal{M}$ and the selection map $\mathcal{F}$ for proportional and tournament selection.

\subsection{The mixing map $\mathcal{M}$}

We now define the mixing map. Following [13], we define

$$
[\text { expr }]= \begin{cases}1 & \text { if expr is true } \\ 0 & \text { otherwise. }\end{cases}
$$

For each integer $i \in[0, n)$, let the $n \times n$ permutation matrix $\sigma_{i}$ be defined by

$$
\left(\sigma_{i}\right)_{u, v}=[u \oplus v=i] .
$$

The matrix $\sigma_{i}$ is symmetric and self-inverse. If $x \in \Lambda$, $\left(\sigma_{i} x\right)_{j}=x_{j \oplus i}$ and if $M$ is an $n \times n$ matrix, $\left(\sigma_{i} M \sigma_{i}\right)_{u, v}=$ $M_{u \oplus i, v \oplus i}$.

If mutation is given by a rate $p$, for each $i \in[0, n)$, define

$$
\mu_{i}=p^{\mathbf{1}^{T} i}(1-p)^{\ell-\mathbf{1}^{T} i}
$$

In general (even when not defined by a rate), mutation is positive whenever $\mu_{i}>0$ for all $i \in[0, n)$.

If we cross parent bit strings $u$ and $v$ using mask $i$, the children are $(u \otimes i) \oplus(v \otimes \bar{i})$ and $(u \otimes \bar{i}) \oplus(v \otimes i)$.

Define $\chi_{i}$ to be the probability that bit string $i$ is used as a crossover mask. For example, for one-point crossover with crossover rate $c$, we have

$$
\chi_{i}= \begin{cases}\frac{c}{\ell-1} & \text { if } i=1,3,7, \ldots, 2^{\ell-1}-1 \\ 1-c & \text { if } i=0 \\ 0 & \text { otherwise. }\end{cases}
$$

Define the $n \times n$ mixing matrix $M$ by

$$
M_{u, v}=\sum_{i, j, k} \mu_{i} \mu_{j} \frac{\chi_{k}+\chi_{\bar{k}}}{2}[((u \oplus i) \otimes k) \oplus((v \oplus j) \otimes \bar{k})=0] .
$$

Note that if mutation is zero, then

$$
M_{u, v}=\sum_{k} \frac{\chi_{k}+\chi_{\bar{k}}}{2}[(u \otimes k) \oplus(v \otimes \bar{k})=0] .
$$

Example. For string length 2, the mixing matrix for one-point crossover with rate $c$ and no mutation is:

$$
M(0)=\left(\begin{array}{cccc}
1 & 1 / 2 & 1 / 2 & 1 / 2-1 / 2 c \\
1 / 2 & 0 & 1 / 2 c & 0 \\
1 / 2 & 1 / 2 c & 0 & 0 \\
1 / 2-1 / 2 c & 0 & 0 & 0
\end{array}\right) .
$$

Finally, define the mixing map $\mathcal{M}$ by

$$
\mathcal{M}(x)_{i}=\left(\sigma_{i} x\right)^{T} M \sigma_{i} x=x^{T}\left(\sigma_{i} M \sigma_{i}\right) x .
$$

The twist $M^{*}$ of $M$ is defined by

$$
M_{i, j}^{*}=M_{i \oplus j, i} .
$$

For example, the twist of the matrix $M(0)$ shown above is:

$$
M(0)^{*}=\left(\begin{array}{cccc}
1 & 1 / 2 & 1 / 2 & 1 / 2-1 / 2 c \\
0 & 1 / 2 & 0 & 1 / 2 c \\
0 & 0 & 1 / 2 & 1 / 2 c \\
0 & 0 & 0 & 1 / 2-1 / 2 c
\end{array}\right)
$$

For zero mutation, $M^{*}$ is upper triangular. This can be derived directly from equation (2) and is stated on page 55 of [13]. 
For later reference, proposition 2.2 of [16] or theorem 6.13 of [13] show that the differential of $\mathcal{M}$ is given by

$$
d \mathcal{M}_{x}=2 \sum_{u} \sigma_{u}^{T} M^{*} \sigma_{u} x_{u}
$$

For more explanation, see one of $[16 ; 13 ; 14 ; 17 ; 15 ; 6]$.

An important property of the mixing map is that $\mathcal{M}$ maps the simplex into itself, and when mutation is positive, $\mathcal{M}$ maps the simplex into its interior [13]. Thus, $\mathcal{G}$ also has this property. This is stated as theorem 4.7 of [13].

\subsection{The selection map $\mathcal{F}$ for proportional se- lection}

For proportional selection, let $F$ denote the diagonal matrix whose diagonal entries are the elements of the fitness vector $f$. Then

$$
\mathcal{F}(x)=\frac{F x}{f^{T} x} .
$$

For later reference, the differential of proportional selection is given by

$$
d \mathcal{F}_{x}=\frac{f^{T} x F-F x f^{T}}{\left(f^{T} x\right)^{2}} .
$$

It is also not hard to see that

$$
\mathbf{1}^{T} d \mathcal{F}_{x}=0
$$

LEMMA 1.

$$
\begin{gathered}
\sigma_{s} d \mathcal{F}_{e_{s}} \sigma_{s}=\frac{1}{f_{s}}\left(\sigma_{s} F \sigma_{s}-e_{0} f^{T} \sigma_{s}\right) \\
\left(\sigma_{s} d \mathcal{F}_{e_{s}} \sigma_{s}\right)_{i, v}= \begin{cases}\frac{f_{i \oplus s}}{f_{s_{s}}} & \text { if } i=v>0 \\
-\frac{f_{i \oplus s}}{f_{s}} & \text { if } i=0 \text { and } v>0 \\
0 & \text { otherwise }\end{cases}
\end{gathered}
$$

In particular, $\sigma_{s} d \mathcal{F}_{e_{s}} \sigma_{s}$ is upper triangular and only nonzero in the upper row and on the diagonal.

\subsection{The selection map $\mathcal{F}$ for tournament selec- tion}

The selection of one individual by $k$-ary tournament selection is done by choosing with replacement a random sample of $k$ individuals (which we will call a tournament) from the population and then selecting the best of these $k$.

To model $k$-ary tournament selection from a population $x \in \Lambda$, we consider the sample space of all unordered $k$ sequences (tournaments) weighted by their probability as determined by $x$. For example, if $k=5$, one such tournament would be $0,0,1,1,1$ which would have probability $\left(\begin{array}{l}5 \\ 2\end{array}\right) x_{0}^{2} x_{1}^{3}$.

In order for a tournament to correspond to the selection of element $i, i$ must be the most fit element in the tournament. Thus, the probability of selecting element $i$ by $k$-ary tournament selection is given by

$$
\mathcal{F}(x)_{i}=\sum_{u=1}^{k}\left(\begin{array}{l}
k \\
u
\end{array}\right) x_{i}^{u}\left(\sum_{j}\left[f_{j}<f_{i}\right] x_{j}\right)^{k-u} .
$$

This formula uses the assumption that fitness is injective, in other words, $u \neq v \Rightarrow f_{u} \neq f_{v}$.
Example: Tournament selection for $\ell=2$ and $k=3$ with fitness function $\langle 4,1,3,2\rangle^{T}$ is given by:

$$
\begin{aligned}
& \mathcal{F}(x)= \\
& {\left[\begin{array}{c}
3 x 0(x 1+x 2+x 3)^{2}+3 x 0^{2}(x 1+x 2+x 3)+x 0^{3} \\
x 1^{3} \\
3 x 2(x 1+x 3)^{2}+3 x 2^{2}(x 1+x 3)+x 2^{3} \\
3 x 3 x 1^{2}+3 x 3^{2} x 1+x 3^{3}
\end{array}\right]}
\end{aligned}
$$

For completness, here is a formula that does not assume injective fitness:

$$
\begin{gathered}
\mathcal{F}(x)_{i}= \\
\left\{\begin{array}{cl}
\sum_{u=1}^{k}\left(\begin{array}{l}
k \\
u
\end{array}\right) x_{i}^{u}\left(\sum_{j}\left[f_{j}<f_{i}\right] x_{j}\right)^{k-u} & \text { if } f_{s}=f_{i} \Rightarrow s=i \\
\sum_{u=1}^{k}\left(\begin{array}{l}
k \\
u
\end{array}\right) x_{i}^{u} \sum_{v=0}^{k-u} \frac{u}{u+v}\left(\begin{array}{c}
k-u \\
v
\end{array}\right) & \\
\left(\sum_{r \neq i}\left[f_{r}=f_{i}\right] x_{r}\right)^{v} & \\
\left(\sum_{j}\left[f_{j}<f_{i}\right] x_{j}\right)^{k-u-v} & \text { otherwise }
\end{array}\right.
\end{gathered}
$$

For the rest of this paper, we assume injective fitness in our analysis of tournament selection. A future paper will analyze non-injective fitness.

Vose [13] models a somewhat different version of tournament selection. Instead of choosing the best element of the tournament, he applies ranking selection to the tournament. His model of ranking selection follows that of Goldberg and Deb [4] in using the integral of a continuously increasing probability density function over the interval $[0,1]$. Goldberg and Deb call this an "assignment function". Ranking selection can be made to select only the best individual by using an assignment function that assigns unit mass to 1 (a translation of the Dirac delta function) as the assignment function. However, when ranking selection is used to select only the best individual, it is not continuous and thus not differentiable. Thus, Vose's results cannot be directly applied to tournament selection as we have defined it above (which we believe is the version most commonly used in practice). In addition, we feel that our model of tournament selection is much simpler and easier to work with.

Lemma 2. For $i=0,1, \ldots, n-1$,

$$
\sum_{j=0}^{n-1} \mathcal{F}(x)_{j}=\left(\sum_{j=0}^{i} x_{j}\right)^{k}
$$

Thus, if $x \in \Lambda, \sum_{j=0}^{n-1} \mathcal{F}(x)_{j}=1$.

Proof Let $\pi$ denote a permutation of $\{0,1, \ldots, n-1\}$ such that $f_{\pi(0)}<f_{\pi(1)}<\ldots<f_{\pi(n-1)}$. Note that equation (5) can be written as

$$
\mathcal{F}(x)_{i}=\sum_{u=1}^{k}\left(\begin{array}{l}
k \\
u
\end{array}\right) x_{i}^{u}\left(\sum_{j=0}^{i-1} x_{\pi(j)}\right)^{k-u}
$$

For $i=0,1, \ldots, n-1$ we prove by induction on $i$ that

$$
\sum_{j=0}^{i} \mathcal{F}(x)_{j}=\left(\sum_{j=0}^{i} x_{\pi(j)}\right)^{k}
$$

For $i=0$, we have $\mathcal{F}(x)_{0}=x_{\pi(0)}^{k}$. 
We assume equation (7) for $i-1$ and prove it for $i$.

$$
\begin{aligned}
\left(\sum_{j=0}^{i} x_{\pi(j)}\right)^{k}= & \left(x_{\pi(i)}+\sum_{j=0}^{i-1} x_{\pi(j)}\right)^{k} \\
= & \sum_{u=0}^{k}\left(\begin{array}{l}
k \\
u
\end{array}\right) x_{\pi(i)}^{u}\left(\sum_{j=0}^{i-1} x_{\pi(j)}\right)^{k-u} \\
& \text { by the binomial theorem } \\
= & \left(\sum_{j=0}^{i-1} x_{\pi(j)}\right)^{k}+\sum_{u=1}^{k}\left(\begin{array}{l}
k \\
u
\end{array}\right) x_{\pi(i)}^{u}\left(\sum_{j=0}^{i-1} x_{\pi(j)}\right)^{k-u} \\
= & \sum_{j=0}^{i-1} \mathcal{F}(x)_{j}+\mathcal{F}(x)_{i}=\sum_{j=0}^{i} \mathcal{F}(x)_{j}
\end{aligned}
$$

This completes the induction proof.

Since we know that for $x \in \Lambda, \sum_{j=0}^{n-1} x_{j}=\sum_{j=0}^{n-1} x_{\pi(j)}=1$, the second formula follows.

The following lemma is straightforward.

LEMMA 3.

$$
\begin{aligned}
& \frac{\partial \mathcal{F}_{i}}{\partial x_{v}}= \\
& \begin{cases}\sum_{u=1}^{k}\left(\begin{array}{l}
k \\
u
\end{array}\right) u x_{i}^{u-1}\left(\sum_{j}\left[f_{j}<f_{i}\right] x_{j}\right)^{k-u} & \text { if } v=i \\
\sum_{u=1}^{k-1}\left(\begin{array}{l}
k \\
u
\end{array}\right) x_{i}^{u}(k-u)\left(\sum_{j}\left[f_{j}<f_{i}\right] x_{j}\right)^{k-u-1}\left[f_{v}<f_{i}\right] & \text { if } v \neq i\end{cases}
\end{aligned}
$$

Lemma 4. For any $x$,

$$
\mathbf{1}^{T} d \mathcal{F}_{x}=k \mathbf{1}^{T}\left(\sum_{j} x_{j}\right)^{k-1}
$$

and for any $x \in \Lambda$,

$$
\mathbf{1}^{T} d \mathcal{F}_{x}=k \mathbf{1}^{T}
$$

Proof Lemma 2 showed that $\sum_{j} \mathcal{F}(x)_{j}=\left(\sum_{j} x_{j}\right)^{k}$. If we differentiate this equation with respect to $x_{i}$, we get:

$$
\sum_{j} \frac{\partial \mathcal{F}_{j}}{\partial x_{i}}=k\left(\sum_{j} x_{j}\right)^{k-1}
$$

Since the right-hand expression is independent of $i$, the first statement of the lemma follows. The second statement is obvious.

Next we evaluate $d \mathcal{F}_{e_{s}}$ where $e_{s}$ denotes the $s^{\text {th }}$ unit vector.

LEMMA 5 .

$$
\left(d \mathcal{F}_{e_{s}}\right)_{i, v}= \begin{cases}k & \text { if } i=v \text { and } f_{s}<f_{v} \\ k & \text { if } i=s \text { and } f_{s} \geq f_{v} \\ 0 & \text { otherwise }\end{cases}
$$

Proof Consider the first case where $i=v$ and $f_{s}<f_{v}$. The first case of formula (9) applies and is nonzero only when $u=1$. In this case, $u x_{i}^{u-1}=1$ since the exponent $u-1$ is zero. The factor $\left(\sum_{j}\left[f_{j}<f_{i}\right] x_{j}\right)^{k-u}=1$ since for $j=s, f_{s}<f_{i}$.

Consider the second case where $i=s$ and $f_{s} \geq f_{v}$. The second case of formula (9) applies and is nonzero only when $u=k-1$. In this case, $x_{i}^{u}=1$ since $i=s$. The factor $\left(\sum_{j}\left[f_{j}<f_{i}\right]\right)^{k-u-1}=1$ since the exponent $k-u-1$ is

One can check that these are the only cases where $\frac{\partial \mathcal{F}_{i}}{\partial x_{v}}\left(e_{s}\right)$ is nonzero.

Corollary 6. For each $s=0,1, \ldots, n-1$,

$$
\left(\sigma_{s} d \mathcal{F}_{e_{s}} \sigma_{s}\right)_{i, v}= \begin{cases}k & \text { if } i=v \text { and } f_{s}<f_{v \oplus s} \\ k & \text { if } i=0 \text { and } f_{s} \geq f_{v \oplus s} \\ 0 & \text { otherwise }\end{cases}
$$

Thus, $\sigma_{s} d \mathcal{F}_{e_{s}} \sigma_{s}$ is upper triangular and has nonzero entries only in the upper row and on the diagonal. Furthermore, it has only a single nonzero entry $k$ in each column.

Proof Recall that $\left(\sigma_{s}\right)_{x, y}=[x \oplus y=s]$, and that $\sigma_{s}$ is symmetric and self-inverse. Further, if $A$ is any $n \times n$ matrix,

$$
\left(\sigma_{s} A \sigma_{s}\right)_{i, v}=A_{i \oplus s, v \oplus s}
$$

Applying this formula to formula (10) gives(11). Upper triangularity is obvious, as is

$$
\mathbf{1}^{T} \sigma_{s} d \mathcal{F}_{e_{s}} \sigma_{s}=k \mathbf{1}^{T}
$$

\subsection{A change of basis}

In this subsection we show how to do an orthonormal change of basis so that we can work in the hyperplane containing the simplex. Recall that $\mathbf{1}$ is the vector of all ones. Note that $\left\{x \in \mathbb{R}^{n}: \mathbf{1}^{T} x=1\right\}$ is the $(n-1)$-dimensional hyperplane that contains the simplex, and $\mathbf{1}^{\perp}=\left\{x \in \mathbb{R}^{n}\right.$ : $\left.\mathbf{1}^{T} x=0\right\}$ is the translate of this hyperplane to the origin.

Lemma 7. Let $A$ be an $n \times n$ real matrix. Suppose that $\mathbf{1}^{T} A=\lambda \mathbf{1}^{T}$ (so that $\mathbf{1}$ is an eigenvector of $A^{T}$ ). Then there is an orthonormal change of basis with basis change matrix $B$ where the first column is $\mathbf{1}$ rescaled to have unit length, and where

$$
B^{T} A B=\left(\begin{array}{cc}
\lambda & 0 \\
* & C
\end{array}\right) .
$$

(Here * denotes possibly nonzero entries.) Then $C$ represents the action of $A$ on the hyperplane $\mathbf{1}^{\perp}=\left\{x \in \mathbb{R}^{n}\right.$ : $\left.\mathbf{1}^{T} x=0\right\}$. The eigenvalues of $A$ in addition to $\lambda$ are the same as the eigenvalues of $C$.

Proof. This proof is adapted from the proof of theorem 6.12 of $[13]$

Since $\mathbf{1}^{T} A=\lambda \mathbf{1}$, it follows that $A: \mathbf{1}^{\perp} \rightarrow \mathbf{1}^{\perp}$. Let $\left\{b_{0}, b_{1}, \ldots, b_{n-1}\right\}$ be an orthonormal basis with $b_{0}$ being $\mathbf{1}$ normalized to have unit length, and let $B$ be the matrix whose columns are this basis. (The Walsh basis is such a basis.) Note that if $j>0$, then

$$
B^{T} A B e_{j}=B^{-1} A b_{j} \subseteq B^{-1}\left(\mathbf{1}^{\perp}\right) \subseteq e_{0}^{\perp} .
$$

(Recall that $e_{j}$ is the $j^{\text {th }}$ unit vector.) Thus, $B^{T} A B$ satisfies equation 12 . 
Observe that $e_{0}^{T} B^{T} A B=\left(B^{T} A \mathbf{1}\right)^{T}=\lambda\left(B^{-1} b_{0}\right)^{T}=\lambda e_{0}^{T}$. Thus, the upper left entry of $B^{T} A B$ is $\lambda$.

With respect to the above basis the elements of $1^{\perp}$ have the form $\left(\begin{array}{l}0 \\ *\end{array}\right)$. Thus, $C$ represents $A$ on $\mathbf{1}^{\perp}$. The eigenvalues of a matrix are invariant under a change of basis.

\subsection{Dynamical system fixed points}

Let $g$ be a map that defines a discrete-time dynamical system. A fixed point $v$ of $g$ is asymptotically stable if there is a neighborhood $U$ of the fixed point such that $\lim _{k \rightarrow \infty} g^{k}(y)=$ $v$ for all $y \in U$. The fixed point $v$ is unstable if there is a neighborhood $U$ of $v$, such that for all $\delta>0$ there exists a point $y$ with $|y-v|<\delta$ such that $g^{k}(y)$ is not in $U$ for some $k^{1}$

A fixed point $v$ is hyperbolic if no eigenvalue of the differential $d g_{v}$ has an eigenvalue with modulus 1. Vose and Eberlein have shown that for proportional selection, the fixed points of $\mathcal{G}$ are hyperbolic for a dense open set of fitness coefficients in the positive orthant [13]. Gedeon, et al. [3] show that for a "typical" mixing operator $\mathcal{G}$ has finitely many fixed points, and Hayes and Gedeon have shown that the fixed points of $\mathcal{G}$ are hyperbolic for a "typical" mixing operator [5].

For a hyperbolic fixed point $v$ of $g$, the asymptotic stability of $v$ is related to the differential $d g_{v}$ at the fixed point. If all eigenvalues of $d g_{v}$ have modulus less than 1 , then $v$ is asymptotically stable. And if any eigenvalue of $d g_{v}$ has modulus greater than 1 , then $v$ is asymptotically unstable. We will define a hyperbolic fixed point to be a saddle fixed point if some eigenvalues have modulus less than 1 and some eigenvalues have modulus greater than 1. Clearly, a saddle fixed point is asymptotically unstable.

The stable manifold theorem (see theorem 10.1 of [9]) characterizes the behavior of $g$ in a sufficiently small neighborhood of a hyperbolic fixed point. If there are $s$ eigenvalues whose modulus is less than 1 , then there is a stable manifold of dimension $s$, and if there are $u$ eigenvalues whose modulus is greater than 1 , then there is an unstable manifold of dimension $u$. The stable manifold consists of points $x$ such that $\lim _{k \rightarrow \infty} g^{k}(x)=v$ and the unstable manifold consists of points $x$ such that $\lim _{k \rightarrow \infty}\left(g^{-1}\right)^{k}(x)=v$ where $g^{-1}$ denotes the inverse of $g$ when the inverse exists. When $g$ is not locally invertible, the unstable manifold can be defined in terms of the past history of its points. See [9] or other books on dynamical systems for details.

Neighborhoods of saddle points can contain trajectories that are eventually repelling. In loose language, there exist initial conditions for which iterations move towards the fixed-point for a time, only to then eventually begin to move away from the fixed-point.

\subsection{Fixed points of the SGA IPM}

The Perron-Frobenius theorem states that there can exist only one fixed-point in the simplex for a linear system with positive irreducible transition matrix [2]. Further, this fixed point is asymptotically stable.

This is exactly the situation for the SGA IPM with proportional selection, positive mutation, and zero crossover since the normalization of proportional selection can be ignored in determining the long-term behavior of the IPM.

\footnotetext{
${ }^{1}$ This definition of unstable is the negation of Lyapunov stability, see [9] for details.
}

Thus, in this situation, $\mathcal{G}$ has one stable fixed point in the interior of the simplex.

Rowe [10] and Richter et al. [8; 7] are example analyses of the Perron-Frobenius situation and movement of the fixedpoint under varying mutation rates.

When the mutation rate is $1 / 2$, the mutation map takes all populations to the center of the simplex. Hence, $\mathcal{G}$ with crossover, any selection method, and any fitness function has the same property.

Vose [13] conjectures that when started at a point in the simplex $\Lambda$, the iterates of $\mathcal{G}$ converge to a fixed point. Wright and Bidwell [19] empirically tested this conjecture and found what appeared to be cyclic behavior. However, these examples used a non-standard mutation which is not bitwise mutation with a rate. Given the extensive experience with GAs, the conjecture seems very likely to be true for bitwise mutation by a rate where the rate is less than or equal to $1 / 2$.

\subsection{Vertex fixed points}

Mask-based crossover is pure in that crossing an individual with itself results only in that individual. Thus, when mutation is zero, the mixing map $\mathcal{M}$ applied to a uniform population of identical individuals gives that population. Selection applied to a uniform population cannot produce any new individuals, and thus the selection map $\mathcal{F}$ applied to a uniform population also gives that population. Thus, we have shown the following:

Lemma 8. Assume no mutation. If $v$ is a vertex of the simplex $\Lambda, \mathcal{G}(v)=v$. In other words, $v$ is a fixed point of $\mathcal{G}$.

The SGA Markov chain model is absorbing when mutation is zero, and the vertex populations are the absorbing states.

For vertex fixed points of $\mathcal{G}$, there are some special results on stability [16], section 11.3 of [13]. A suitable rearrangement of the rows and columns of the differential of $\mathcal{G}$ is upper triangular, so the eigenvalues are the diagonal elements. In this section we derive formulas for the eigenvalues for proportional and tournament selection. For proportional selection, these are from [16] and theorem 11.8 of [13]. For tournament selection as we have defined it, these results are new.

$$
\begin{aligned}
& \text { Lemma } 9 . \text { For } x \in \Lambda \text {, } \\
& \qquad \mathbf{1}^{T} d \mathcal{G}_{x}=0 \text { for proportional selection } \\
& \mathbf{1}^{T} d \mathcal{G}_{x}=2 k \mathbf{1}^{T} \text { for tournament selection }
\end{aligned}
$$

Proof Theorem 6.13 of [13] shows $\mathbf{1}^{T} d \mathcal{M}_{x}=2\left(\sum x_{u}\right) \mathbf{1}^{T}$. Thus, if $x \in \Lambda, \sum x_{u}=1$, so $\mathbf{1}^{T} d \mathcal{M}_{x}=2 \mathbf{1}^{T}$. Applying the chain rule, $\mathbf{1}^{T} d \mathcal{G}_{x}=\mathbf{1}^{T} d \mathcal{M}_{\mathcal{F}(x)} d \mathcal{F}_{x}=2 \mathbf{1}^{T} d \mathcal{F}_{x}$. We now apply equation (4) for proportional selection and lemma 4 for tournament selection.

LEMma 10. Let $\mathcal{F}$ be the heuristic function for either proportional or tournament selection. For $s=0,1, \ldots, n-1$,

$$
\sigma_{s} d \mathcal{G}_{e_{s}} \sigma_{s}=2 M^{*} \sigma_{s} d \mathcal{F}_{e_{s}} \sigma_{s}
$$

If mutation is zero, $\sigma_{s} d \mathcal{G}_{e_{s}} \sigma_{s}$ is upper triangular and the eigenvalues are the diagonal elements. 
Proof First, note that $\mathcal{F}\left(e_{s}\right)=e_{s}$ since $e_{s}$ is a population consisting only of individuals equal to $s$, and any such population is fixed under selection.

$$
\begin{aligned}
d \mathcal{G}_{e_{s}} & =d \mathcal{M}_{\mathcal{F}\left(e_{s}\right)} d \mathcal{F}_{e_{s}} \quad \text { by the chain rule } \\
& =d \mathcal{M}_{e_{s}} d \mathcal{F}_{e_{s}} \\
& =2 \sum_{u} \sigma_{u} M^{*} \sigma_{u}\left(e_{s}\right)_{u} d \mathcal{F}_{e_{s}} \\
& =2 \sigma_{s} M^{*} \sigma_{s} d \mathcal{F}_{e_{s}}
\end{aligned}
$$

Thus, $\sigma_{s} d \mathcal{G}_{e_{s}} \sigma_{s}=2 M^{*} \sigma_{s} d \mathcal{F}_{e_{s}} \sigma_{s}$. Recall that zero mutation implies that $M^{*}$ is upper triangular. Upper triangularity follows since both $M^{*}$ and $\sigma_{s} d \mathcal{F}_{e_{s}} \sigma_{s}$ are upper triangular.

LEMMA 11. Assume zero mutation and $s=0,1, \ldots, n-1$. If $\mathcal{F}$ is the heuristic function for tournament selection.

$$
\left(\sigma_{s} d \mathcal{G}_{e_{s}} \sigma_{s}\right)_{i, v}= \begin{cases}2 k M_{i \oplus v, i} & \text { if } f_{s}<f_{v \oplus s} \\ 2 k M_{0, v} & \text { if } f_{s} \geq f_{v \oplus s} \\ 0 & \text { otherwise }\end{cases}
$$

\section{Proof}

$$
\begin{aligned}
& \left(\sigma_{s} d \mathcal{G}_{e_{s}} \sigma_{s}\right)_{i, v} \\
& =2 \sum_{u} M_{i, u}^{*}\left(\sigma_{s} d \mathcal{F}_{e_{s}} \sigma_{s}\right)_{u, v} \\
& =2 \sum_{u} M_{i, u}^{*} k\left[\left((u=v) \wedge\left(f_{s}<f_{v \oplus s}\right)\right) \vee\left((u=0) \wedge\left(f_{s} \geq f_{v \oplus s}\right)\right)\right] \\
& = \begin{cases}2 k M_{i, v}^{*}=2 k M_{i \oplus v, i} & \text { if } f_{s}<f_{v \oplus s} \\
2 k M_{i, 0}^{*}=2 k M_{v, 0}=2 k M_{0, v} & \text { if } f_{s} \geq f_{v \oplus s} \\
0 & \text { otherwise }\end{cases}
\end{aligned}
$$

Theorem 12. Assume tournament selection. If mutation is zero and $s=0,1, \ldots, n-1$, the eigenvalues of $d \mathcal{G}_{e_{s}}$ considered as a map from $\mathbf{1}^{\perp}$ to itself are

$$
\left\{2 k M_{0, v}\left[f_{s}<f_{v \oplus s}\right]: v=1,2, \ldots, n-1\right\} .
$$

Proof The eigenvalues of $d \mathcal{G}_{e_{s}}$ are the same as the eigenvalues of $\sigma_{s} d \mathcal{G}_{e_{s}} \sigma_{s}$. Thus, the eigenvalues of $d \mathcal{G}_{e_{s}}$ are given by the first case of equation (13) when $f_{s}<f_{v \oplus s}$ and $i=v$, and are zero when $f_{s} \geq f_{v \oplus s}$.

Lemma 9 shows that $\mathbf{1}$ is an eigenvector of $d \mathcal{G}_{e_{s}}^{T}$, so lemma 7 applies. Since the matrix $B$ of lemma 7 (which can be the Walsh basis) is orthonormal, the eigenvalues of $d \mathcal{G}_{e_{s}}$ considered as a mapping from $\mathbf{1}^{\perp}$ to itself are the same as the eigenvalues of $d \mathcal{G}_{e_{s}}$ applied to $\mathbb{R}^{n}$ less the eigenvalue $2 k M_{0,0}=2 k$.

THEOREM 13. Assume proportional selection. If mutation is zero and $s=0,1, \ldots, n-1$, the eigenvalues of $d \mathcal{G}_{e_{s}}$ considered as a map from $\mathbf{1}^{\perp}$ to itself are

$$
\left\{\frac{f_{v \oplus s}}{f_{s}} M_{0, v}: v=1,2, \ldots, n-1\right\} .
$$

Proof This theorem states the result of theorem 3.5 of [16] as extended by the results of section 4 of the same paper. It is also stated as one part of theorem 11.8 of [13]. It can be proved directly by combining lemma 1 with lemma 10, and then applying lemma 7 .

If $i \in(0, n)$, let $\mathrm{hi}(i)$ and $\operatorname{lo}(i)$ be the smallest and largest $k$ such that $i \otimes 2^{k} \neq 0$. In other words, $\operatorname{lo}(i)$ is the position of the leftmost one in the binary representation of $i$, and hi $(i)$ is the position of the rightmost one in the binary representation of $i$. Let $\delta(i)=\operatorname{hi}(i)-\operatorname{lo}(i)+1 ; \delta(i)$ is commonly called the defining length of $i$.

Lemma 14. For one-point crossover with crossover rate $c$ and zero mutation,

$$
M_{0, v}=\frac{1}{2}\left(1-c+c \frac{\ell-\delta(v)}{\ell-1}\right) .
$$

For uniform crossover with crossover rate $c$ and zero mutation,

$$
M_{0, v}=\frac{1}{2}\left(1-c+c 2^{1-\mathbf{1}^{T} i}\right)
$$

Proof. By equation (2) $M_{0, v}=\sum_{u} \frac{\chi_{u}+\chi_{\bar{u}}}{2}[u \otimes v=0]$. The formulas follow by direct computation. More details for proof of the uniform crossover formula are given in the proof of lemma 5.2 of [16].

COROllary 15. Assume tournament selection. Let $c$ denote the crossover rate for one-point or uniform crossover. For one-point crossover, the spectrum of $d \mathcal{G}_{e_{k}}$, considered as a map from $\mathbf{1}^{\perp}$ to itself, is given by

$$
\begin{aligned}
& \operatorname{spec}\left(d \mathcal{G}_{e_{s}}\right)= \\
& \left\{k\left(1-c+c^{\ell} \frac{\ell-\delta(i)}{\ell-1}\left[f_{v}<f_{v \oplus s}\right]\right): i=1, \ldots, n-1\right\} .
\end{aligned}
$$

For uniform crossover, the spectrum of $d \mathcal{G}_{e_{k}}$ is given by

$$
\begin{aligned}
& \operatorname{spec}\left(d \mathcal{G}_{e_{s}}\right)= \\
& \left\{k\left(1-c+c 2^{1-\mathbf{1}^{T} i}\right)\left[f_{v}<f_{v \oplus s}\right]: i=1, \ldots, n-1\right\} .
\end{aligned}
$$

Corollary 16. Assume proportional selection. Let c denote the crossover rate for one-point or uniform crossover. For one-point crossover, the spectrum of $d \mathcal{G}_{e_{k}}$, considered as a map from $\mathbf{1}^{\perp}$ to itself, is given by

$\operatorname{spec}\left(d \mathcal{G}_{e_{s}}\right)=\left\{\frac{f_{i \oplus s}}{f_{s}}\left(1-c+c^{\ell} \frac{\ell-\delta(i)}{\ell-1}\right): i=1, \ldots, n-1\right\}$.

For uniform crossover, the spectrum of $d \mathcal{G}_{e_{k}}$ is given by

$$
\operatorname{spec}\left(d \mathcal{G}_{e_{s}}\right)=\left\{\frac{f_{i \oplus s}}{f_{s}}\left(1-c+c 2^{1-\mathbf{1}^{T} i}\right): i=1, \ldots, n-1\right\} .
$$

Proof. This corollary is a restatement of theorem 3.4 and lemma 5.1 of [16]. It also follows directly from theorem 13 and lemma 14.

For an example of the application of this theorem, see subsection 5.1 .

Note that corollary 16 shows that each eigenvalue of the differential of $\mathcal{G}$ at vertex fixed point $e_{k}$ corresponds to the fitness of some other search space point $i \oplus k$. More precisely, it is the fitness ratio $\frac{f_{i \oplus k}}{f_{k}}$ times a factor that depends on $i$ and on the crossover method. 
For a crossover rate of zero (pure selection), only the vertices corresponding to global optima are stable fixed points. Global optima with no globally optimal neighbors are asymptotically stable. As the crossover rate increases, more vertices may become stable fixed points. But vertices corresponding to search space points with more fit neighbors can never be stable.

The following lemma shows that stability in $\mathbb{R}^{n}$ for vertex fixed points is the same as stability in the simplex.

LEMMA 17. If $v$ is a vertex fixed point with the spectral radius (modulus of largest eigenvalue) of $d \mathcal{G}_{v}$ greater than 1 , then $v$ is an asymptotically unstable fixed point of $\mathcal{G}$ considered as a map from the hyperplane containing the simplex to itself.

Proof. This is theorem 4.3 of [16]. Or lemma 7 can be used to prove this theorem.

\subsection{Bistability}

In dynamical systems theory, bistability refers to a situation where there are two stable fixed points with distinct domains of attraction. The fixed point that the system converges to depends on the initial conditions.

It is easy to construct fitness functions with multiple peaks (local maxima) so that the SGA IPM with no mutation has stable fixed points which are the uniform populations consisting only of multiple copies of the fitness peaks. See theorems 15 and 16 for details. (Note, however, that a local maximum of the fitness function does not necessarily correspond to a stable fixed point.) It is one of the main results of this paper (see theorem 31) that with a sufficiently small increase in mutation, these fixed points move inside the simplex.

Thus, it is easy to construct examples where the SGA IPM has dynamical systems bistability, and these examples are not surprising. However, there is a more restricted form of bistability that can happen in infinite population models which is surprising.

When the mutation rate is $1 / 2$, then there is a single stable fixed point of the SGA IPM at the center of the simplex. One might guess that if there was a single fitness peak, then as the mutation rate decreased from $1 / 2$ to zero, this stable fixed point would migrate from the center of the simplex to the uniform population corresponding to the fitness peak. However, something more complex can happen [23]: at a critical mutation rate, the stable fixed point bifurcates into two stable fixed points and one unstable fixed point. As the mutation rate continues to decrease, one stable fixed point move toward the fitness peak, the other stays near the center of the simplex, with the unstable fixed point between them. At another smaller critical mutation rate, the unstable fixed point joins with the one of the stable fixed points, and both of these fixed points disappear. Thus, at a range of mutation rates between the two critical mutation rates, there are two stable fixed points which is bistability in the sense defined above. But it is surprising since the bistability is not caused by multiple fitness peaks, but is rather related to the disruptiveness of the combination of mutation and crossover. This situation with two stable fixed points and one fitness peak was called bistability in [23]; clearly this is a more restricted form of bistability than the dynamical system definition mentioned above.

The above description is based on the gene pool model given in [23]. Gene pool crossover takes the population di- rectly to linkage equilibrium in one step. In this model, the fixed point equation reduces to an equation in a single variable for the NEEDLE (needle-in-the-haystack) and BINEEDLE fitness functions, experiments in running the SGA IPM with conventional two parent crossover show very similar bistability behavior on these fitness functions. Below are the formulations for NEEDLE and BINEEDLE.

$$
\begin{gathered}
N E E D L E(x)= \begin{cases}1+a & \text { all ones string } \\
1 & \text { otherwise }\end{cases} \\
B I N E E D L E(x)= \begin{cases}1+a & \text { all ones string } \\
1 & \text { otherwise } \\
1+a & \text { all zeros string }\end{cases}
\end{gathered}
$$

In practical terms, when there is bistability, a GA initialized with a random population is likely to be trapped close to the center of the simplex stable fixed point which prevents it from accumulating points of the optimal population. This is illustrated by the results of Suzuki and Iwasa [11] on the NEEDLE fitness (which they called a "babel-like fitness landscape"). They found that crossover accelerated time to convergence if the crossover rate was not too high, but over a critical crossover rate, the time to find the needle diverged. The discovery of the bistability phenomenon explained their results.

Bistability (in the more restricted sense) is only known to occur in the presence of crossover, mutation, and selection. This more restricted bistability phenomenon was discovered by Boerjlist et al [1] in a quasi species model of virus reproduction, and was analyzed for the NEEDLE and the BINEEDLE fitness functions and proportional selection in [23]. These results were extended to truncation selection in [20] and to a "sloping plateau" fitness function in [21], all by using the gene pool model.

When bistability occurs, there is one fixed point "close" to the fitness peak, and one fixed point "close" to the center of the simplex. For the NEEDLE fitness functions, the fitness peak is at a vertex of the simplex. Thus, when the GA infinite population model is initialized with a population corresponding to the center of the simplex, the GA model will converge to the center of the simplex fixed point, and when the model is initialized with a population corresponding to the fitness peak, the model will converge to the fitness peak fixed point. A finite population GA with the same parameters and fitness function, when initialized with a random population will likely be "trapped" for a long time by the center of the simplex fixed point, and while when initialized at the fitness peak, will likely be "trapped" for a long time by the fitness peak fixed point. ("A long time" is the best that we can say since the GA with mutation is an ergodic Markov chain, and all populations will be visited infinitely often.)

\section{QUESTIONS}

The dichotomy of many fixed points for crossover-selection GAs and one fixed point for mutation-selection GAs and for mutation rate $1 / 2$ suggests some questions:

- What happens to the vertex fixed points when mutation is increased slightly from zero? We answer this question for hyperbolic fixed points in the next section. 
- What happens to the vertex fixed points when the mutation rate is increased from 0 to $1 / 2$ ? Recall that when the mutation rate is $1 / 2$, there is a single stable fixed point at the center of the simplex.

- What happens to the Perron-Frobenius fixed point as the crossover rate is increased from zero? Presumably, the fixed point must bifurcate when there are multiple stable fixed points under crossover. What kinds of bifurcations are possible?

\section{THE MOVEMENT OF VERTEX FIXED POINTS UNDER SMALL POSITIVE MU- TATION}

In this section we investigate the behavior of hyperbolic vertex fixed points of $\mathcal{G}$ as the mutation rate increases from zero. Let $p$ denote the mutation rate, and let $\mathcal{G}: \Lambda \times$ $(-1,1) \rightarrow \Lambda$ denote the SGA map parametrized by the mutation rate $p$. (While a negative mutation rate is not meaningful in terms of a GA, the formulas for $\mathcal{G}$ apply for a negative mutation rate, and this allows us to not consider one-sided derivatives.) Let $v=v_{0}$ be a hyperbolic vertex fixed point for $\mathcal{G}(x, 0)$. We will use the notation $\mathcal{G}_{p}(x)$ for $\mathcal{G}(x, p)$ when it is convenient.

While we are stating our results in term of the mutation rate, in fact all we need is that $\mathcal{G}(x, p)$ is continuously (i. e. $C^{1}$ ) differentiable in both $x$ and $p$, and $\mathcal{G}_{p}$ maps the simplex into its interior for $p>0$.

In this section, we will be differentiating with respect to both $x$ and $p$, so we will use a different notation for these derivatives. Let $\frac{\partial \mathcal{G}}{\partial x}(y, q)$ denote the derivative of $\mathcal{G}$ with respect to $x \in \mathbb{R}^{n}$ evaluated at $(y, q) \in \mathbb{R}^{n} \times(-1,1)$. (In the notation of the previous section, this was $d\left(\mathcal{G}_{p}\right)_{(y, q)}$.) Let $\frac{\partial \mathcal{G}}{\partial p}$ denote the derivative of $\mathcal{G}$ with respect to the mutation rate $p$.

Let $i d: \mathbb{R}^{n} \rightarrow \mathbb{R}^{n}$ denote the identity map. Of course, the differential of $i d$ is the identity matrix.

LEMma 18. If $g: \mathbb{R}^{n} \rightarrow \mathbb{R}^{n}$ is differentiable at a point $y$ with no eigenvalues equal to 1 , then $g-i d$ is differentiable with a nonsingular differential at $y$.

Proof. There is a similarity transformation $P$ such that $P^{-1} \frac{d g}{d x}(y) P$ is in Jordan canonical form with the eigenvalues on the diagonal. Then $P^{-1}\left(\frac{d g}{d x}(y)-I\right) P$ is also in Jordan canonical form with eigenvalues on the diagonal. Thus, the eigenvalues of $\frac{d g}{d x}(y)-I$ are the eigenvalues of $\frac{d g}{d x}(y)$ minus 1 , and by assumption these are nonzero. Thus, $\frac{d g}{d x}(y)-I$ is nonsingular.

Define $\mathcal{H}: \mathbb{R}^{n} \times(-1,1) \rightarrow \mathbb{R}^{n}$ by $\mathcal{H}=\mathcal{G}-i d$. Recall that we have assumed that $v$ is a hyperbolic vertex fixed point of $\mathcal{G}$ which means that $\frac{\partial \mathcal{G}}{\partial x}(p, 0)$ has no eigenvalues on the unit circle. Thus, $\mathcal{H}(v, 0)=0$ and $\frac{\partial \mathcal{H}}{\partial x}(v, 0)=\frac{\partial \mathcal{G}}{\partial x}(v, 0)-I$ is non-singular by Lemma 18.

LEMmA 19. There is a neighborhood $V$ of $v$, an $\varepsilon>0$, and a continuously differentiable function $h:[0, \varepsilon) \rightarrow V$ such that $\mathcal{H}(h(p), p)=0$ and $h(0)=v$. If we define $v_{p}=$ $h(p)$, then $\mathcal{G}\left(v_{p}, p\right)=v_{p}$, so $v_{p}$ is a fixed point of $\mathcal{G}_{p}$. Furthermore, the derivative of $h$ is given by

$$
\frac{\partial h}{\partial p}(p)=-\left(\frac{\partial \mathcal{H}}{\partial x}(h(p), p)\right)^{-1} \frac{\partial \mathcal{H}}{\partial p}(h(p), p) .
$$

Proof. By the above argument our assumption of hyperbolicity of the vertex fixed point $v$ implies that $\frac{\partial \mathcal{H}}{\partial x}(v, 0)$ is nonsingular. There is a neighborhood $U$ of $v$ and a $\delta>0$ such that $\frac{\partial \mathcal{H}}{\partial x}(x, p)$ is nonsingular for $x \in U$ and $p \in(-\delta, \delta)$. The implicit function theorem shows that there is a neighborhood $V \subseteq U$ of $v$, an $\varepsilon>0$, and a function $h$ with the required properties.

We have now shown that as $p$ increases from 0 , there is a path of fixed points $v_{p}$ of $\mathcal{G}_{p}$. This path can intersect the boundary of the simplex only at $v=v_{0}$ since $\mathcal{G}_{p}$ maps the boundary of the simplex into the interior of the simplex for $p>0$.

\subsection{Asymptotically stable fixed vertex points}

LEMma 20. Let $v$ be a hyperbolic asymptotically stable vertex fixed point of $\mathcal{G}_{0}$. Then there is a neighborhood $V$ of $v$ in $\Lambda$ and $a \delta>0$ such that for all $p<\delta, \mathcal{G}_{p}$ has a unique fixed point in $V$, and this fixed point is asymptotically stable.

Proof. Taking standard matrix norm in the eigenvector basis $\|\cdot\|$ we have $\left\|\frac{\partial \mathcal{G}}{\partial x}(v, 0)\right\|=\alpha<1$. By continuity of the derivative, there exists a $\beta>\alpha$, a small neighborhood $U \subset \Lambda$ of $v$, and a $\delta>0$ such that for all $(y, p) \in U \times[0, \delta)$ we have

$$
\left\|\frac{\partial \mathcal{G}}{\partial x}(y, p)\right\| \leq \beta<1
$$

By going to a smaller neighborhood $V \subseteq U$ if necessary, we have that for any $p \in[0, \delta)$ and any pair $y, z \in V$,

$$
\begin{aligned}
\left\|\mathcal{G}_{p}(y)-\mathcal{G}_{p}(z)\right\| & =\left\|\frac{\partial \mathcal{G}}{\partial x}(y)\right\|\|y-z\|+o(y-z) \\
& \leq \beta\|y-z\|+\frac{1-\beta}{2}\|y-z\| \\
& \leq \frac{1+\beta}{2}\|y-z\| .
\end{aligned}
$$

Thus, $\mathcal{G}_{p}$ is a contraction on $V$ for all $p \in[0, \delta)$. By the contraction mapping theorem (theorem 2.5 of [9]), there is a unique fixed point $v_{p} \in V$ for all $p \in[0, \delta)$.

Our primary theorem on the movement of stable fixed points, theorem 31 is postponed to subsection 4.3

\subsection{Unstable fixed vertex points}

THEOREM 21. Let $v=v_{0}$ be a hyperbolic unstable vertex fixed point of $\mathcal{G}_{0}$. Then for sufficiently small $p>0, \mathcal{G}_{p}$ has no fixed point in $\Lambda$.

Proof. The domain of this proof is the $(n-1)$-dimensional hyperplane $H=\left\{x \in \mathbb{R}^{n}: \mathbf{1}^{T} x=1\right\}$. We can apply lemma 7 to represent $\frac{\partial \mathcal{G}}{\partial x}\left(v_{0}, 0\right)$ in the hyperplane $\mathbf{1}^{\perp}=\left\{x \in \mathbb{R}^{n}\right.$ : $\left.\mathbf{1}^{T} x=0\right\}$, and when we refer to differentials in this proof, we are referring to their representation in $\mathbf{1}^{\perp}$.

The implicit function theorem argument of lemma 19 shows that there is a $\xi>0$ such that $v_{p}$ is a hyperbolic fixed point of $\mathcal{G}(x, p)$ for $p \in(-\xi, \xi)$.

By applying the Center Manifold theorem [9] to the map $\mathcal{H}: H \times(-\xi, \xi) \rightarrow H \times(-\xi, \xi)$ defined by $\mathcal{H}(x, p)=(\mathcal{G}(x, p), p)$, we conclude that the stable manifold $W^{s}\left(v_{p}, \mathcal{G}(x, p)\right)$ and the unstable manifold $W^{u}\left(v_{p}, \mathcal{G}(x, p)\right)$ depend $C^{1}$ jointly on 
both $x$ and $p$. In other words, there exists a $\delta>0$ with $\delta<\xi$ such that for each such $p \in(-\delta, \delta)$ the unstable manifold can be represented as a graph of a $C^{1}$ function

$$
\sigma: E_{\left(v_{0}, 0\right)}^{u} \times(-\delta, \delta) \rightarrow E_{\left(v_{0}, 0\right)}^{s}
$$

where $E_{\left(v_{0}, 0\right)}^{u}$ is a span of the eigenvectors that correspond to eigenvalues of $\frac{\partial \mathcal{G}}{\partial x}\left(v_{0}, 0\right)$ with modulus greater than 1 and where $E_{\left(v_{0}, 0\right)}^{s}$ is a span of the eigenvectors that correspond to eigenvalues of $\frac{\partial \mathcal{G}}{\partial x}\left(v_{0}, 0\right)$ with modulus less than 1 . The graph consists of triples $(x, p, \sigma(x, p))$ where $x \in E_{\left(x_{0}, 0\right)}^{u}$, $p \in(-\delta, \delta)$ and $\sigma(x) \in E_{\left(x_{0}, 0\right)}^{s}$.

Suppose that $v_{p}$ for $p \in(0, \delta)$ lies in the interior of $\Lambda$. Since $v_{0}$ hyperbolic and unstable, $\frac{\partial \mathcal{G}}{\partial x}\left(v_{0}, 0\right)$ has at least one eigenvalue with modulus greater than 1 . Thus, the unstable manifold $W^{u}\left(v_{0}, \mathcal{G}(x, 0)\right)$ is nonempty. The graphs of $W^{u}\left(v_{0}, \mathcal{G}(x, 0)\right)=\sigma(0)$ and $W^{u}\left(v_{0}, \mathcal{G}(x, p)\right)=\sigma(p)$ are $C^{1}$ close to each other and have the same dimension.

Let $B_{r}^{u}\left(v_{0}\right)$ be a ball of radius $r$ in $E_{\left(v_{0}, 0\right)}^{u}$. For any $\varepsilon>0$ (where $\varepsilon$ will be chosen later), there exists an $r>0$ and an $\eta$ with $0<\eta<\delta$ such that

$$
\|\sigma(x, p)-\sigma(x, 0)\|_{C^{1}}<\varepsilon \text { for all } x \in B_{r}^{u}\left(v_{0}\right), \quad p \in(0, \eta) .
$$

Since $v_{0}$ is a vertex of $\Lambda$ and $E_{\left(v_{0}, 0\right)}^{u}$ is a linear space, we must have that

$$
B_{r}^{u}\left(v_{0}\right) \cap \operatorname{ext}(\Lambda) \neq \emptyset, \quad \text { for all } r
$$

where $\operatorname{ext}(\Lambda)$ is the exterior of $\Lambda$. Since $W_{\left(v_{0}, 0\right)}^{u}$ is tangent to $E_{\left(v_{0}, 0\right)}^{u}$ for sufficiently small $r$ and $y \in B_{r}^{u}\left(v_{0}\right) \backslash\left\{v_{0}\right\}$, we must have

$$
\operatorname{graph}(\sigma(y, 0)) \cap \operatorname{ext}(\Lambda) \neq \emptyset .
$$

Let $y_{0} \in \operatorname{graph}(\sigma(y, 0)) \cap \operatorname{ext}(\Lambda)$. Then there is a positive distance $\epsilon$ between the compact set $\operatorname{graph}\left(\sigma\left(y_{0}, 0\right)\right)$ and $\Lambda$. Therefore, by equation (19)

$$
\operatorname{graph}\left(\sigma\left(y_{0}, p\right)\right) \cap \operatorname{ext}(\Lambda) \neq \emptyset
$$

for all $p \in(0, \eta)$. Let $y_{p}$ be in this set.

The reverse iterates of $y_{p}$ under $\mathcal{G}_{p}$ must converge to $v_{p}$. Thus, there must be some reverse iterate of $y_{p}$ that is in $\Lambda$ but is mapped by $\mathcal{G}_{p}$ to $\operatorname{ext}(\Lambda)$. But this contradicts that fact that $\mathcal{G}_{p}$ must map $\Lambda$ to the interior of $\Lambda$. Thus, our assumption that $v_{p} \in \Lambda$ is not correct.

\subsection{The direction of movement of fixed points as mutation increases from zero}

Recall that the function $h$ defined in lemma 19 defined the local path of fixed points as the mutation rate $p$ increased from zero. In this section we calculate $\frac{d h}{d p}$ and discuss the implications of the result.

Throughout this section $v$ will be a vertex fixed point of $\mathcal{G}_{0}$. Without loss of generality, we can rearrange the order of the coordinates of $\mathbb{R}^{n}$ so that $v=e_{0}$ which is the first unit vector in $\mathbb{R}^{n}$.

LEMma 22. Let $v$ be a vertex fixed point and assume that mutation is bitwise mutation with mutation rate $p$. Then

$$
\frac{\partial \mathcal{G}}{\partial p}(v, 0)= \begin{cases}-\ell & \text { if } i=0 \\ 1 & \text { if } \mathbf{1}^{T} i=1 \\ 0 & \text { otherwise }\end{cases}
$$

Furthermore, the vector $\frac{\partial \mathcal{G}}{\partial p}(v, 0)-v$ is in the direction of the simplex.
Proof. The GA map $g$ can be written as a composition of a selection map, a crossover map, and a mutation map. Thus,

$$
\mathcal{G}(p, x)=\mathcal{U}(\mathcal{C}(\mathcal{F}(x, p))) .
$$

Since the crossover map $\mathcal{C}$ and the selection map $\mathcal{F}$ don't depend on the mutation rate $p$, and since $v$ is a fixed point of $\mathcal{C}$ and $\mathcal{F}$, we have that $\frac{\partial \mathcal{G}}{\partial p}(v, 0)=\frac{d \mathfrak{u}}{\partial p}(v, 0)$.

The definition of the mutation map is

$$
\mathcal{U}(x, p)=A x .
$$

where $A$ is the $n \times n$ matrix defined by

$$
A_{i, j}=p^{\mathbf{1}^{T}(i \oplus j)}(1-p)^{\ell-\mathbf{1}^{T}(i \oplus j)} .
$$

Thus,

$$
\mathcal{U}(p, x)_{i}=\sum_{j} p^{\mathbf{1}^{T}(i \oplus j)}(1-p)^{\ell-\mathbf{1}^{T}(i \oplus j)} x_{j} .
$$

Since $v$ is the first unit vector in $\mathbb{R}^{n}$,

$$
\mathcal{U}(p, v)_{i}=p^{\mathbf{1}^{T} i}(1-p)^{\ell-\mathbf{1}^{T} i} .
$$

Clearly, for $\mathbf{1}^{T} i \notin\{0,1\}, \frac{\partial u}{\partial p}(v, 0)_{i}=0$, and it is easy to check that for $\mathbf{1}^{T} i=1, \frac{\partial u}{\partial p}(v, 0)_{i}=1$ and $\frac{\partial u}{\partial p}(v, 0)_{0}=-\ell$. Thus, we have shown that equation (20) holds.

Define the change-of-basis matrix $P$ to have columns $e_{0}, e_{1}-$ $e_{0}, e_{2}-e_{0}, \ldots, e_{n-1}-e_{0}$. Note that the columns of $P$, except for the first, are the directions from $v=e_{0}$ to the other vertices of the simplex. Multiplying by $P$ transforms the standard basis into a basis whose elements are the columns of $P$. A vector from $v$ points into the simplex if it lies in the plane of the simplex (i. e., the sum of the coordinates are zero) and if its coordinates after the first in this basis are nonnegative. Clearly, $\frac{\partial g}{\partial p}(v, 0)$ satisfies these conditions.

Recall that in section 2 we defined the twist $A^{*}$ of an $n \times n$ matrix $A$ to have $i, j$ th entry $A_{i \oplus j, i}$. A matrix $A$ is called separative if $A_{i, j} \neq 0$ implies that $i^{T} j=0$.

Mixing (crossover and mutation) is defined through a mixing matrix $M(p)$ which we parametrize by the mutation rate $p$. See subsection 2.1 for more details.

LEMma 23. The mixing matrix $M(0)$ (for zero mutation) is separative.

Proof. Theorems 6.5 and 6.6 of [13] show that $M(0)$ is separative.

If matrix $A$ is separative, then its twist $A^{*}$ satisfies the condition

$$
A_{i, j}^{*} \neq 0 \Longrightarrow i=i \otimes j
$$

We will call a matrix satisfying this condition twist separative. Clearly, $M(0)^{*}$ in the example above is twist separative.

For example, suppose that $i=6=110$ and $j=3=011$. Then $i \otimes j=010 \neq 110=i$, so if $\mathrm{A}$ is twist separative, $A_{6,3}=0$. So whenever there is a locus of $j$ which is zero while the corresponding locus of $i$ is one, the pair $i, j$ does not satisfy $i=i \otimes j$, and thus $A_{i, j}=0$.

LemMa 24. A twist separative matrix is upper triangular. 
Proof. $i=i \otimes j \Rightarrow i \leq j$.

LEMMA 25. The inverse of a twist separative matrix is twist separative.

Proof. The following is a "back-substitution" algorithm to compute the inverse of an upper triangular matrix:

INVERSEUPPERTRIANGULAR $(A)$

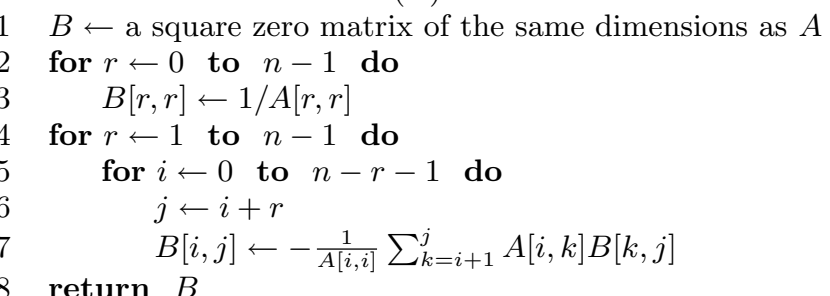

Let $i, j$ be such that $i \neq i \otimes j$. We need to show that $B[i, j]=0$. As above, there must be a locus at which $j$ has a zero bit while $i$ has a one bit. Consider the possibilities for that locus of the summation index $k$ in line 7 of the algorithm. If that locus of $k$ is a one bit, then $k \neq k \otimes j$ which implies that $B[k, j]=0$. If that locus of $k$ is a zero bit, then $i \neq i \otimes k$ which implies that $A[i, k]=0$. Thus, each term of the summation of line 7 is zero, which implies that $B[i, j]=0$.

Lemma 26. If $A$ is an $n \times n$ twist separative matrix and $B=A^{-1}$, then for $j$ such that $\mathbf{1}^{T} j=1, B_{0, j}=\frac{A_{0, j}}{A_{0,0} A_{j, j}}$. is

Proof. Line 7 of the above algorithm applied to $B[0, i]$

$$
B[0, j] \leftarrow-\frac{1}{A[0,0]} \sum_{k=1}^{j} A[0, k] B[k, j] .
$$

Since $B$ is twist separative, $B[k, j]=0$ for $k=1,2, \ldots, j-1$. Thus, the only nonzero term in the summation is $A[0, j] B[j, j]=$ $A[0, j] / A[j, j]$.

Leмma 27. The product of twist separative matrices is twist separative.

Proof. The following is the algorithm to compute the product of upper triangular matrices:

Productuppertriangular $(A, B)$

$1 C \rightarrow$ a square matrix of the same dimensions as $A$ and $B$

for $i \leftarrow 0$ to $n-1$ do

for $j \leftarrow i$ to $n-1$ do

return $C$$$
C[i, j] \leftarrow \sum_{k=i}^{j} A[i, k] B[k, j]
$$

The argument of the proof of Lemma 25 applies to line 4 of this algorithm to show that $i \neq i \otimes j \Rightarrow C[i, j]=0$.

Lemma 28. The differential of $\mathcal{G}$ at simplex vertex $v=e_{0}$ is given by

$$
\frac{\partial \mathcal{G}}{\partial x}(v, p)=\frac{\partial \mathcal{G}}{\partial x}\left(e_{0}, p\right)=2 M(p)^{*} \frac{d \mathcal{F}}{d x}\left(e_{0}\right) .
$$

where $\mathcal{F}$ denotes the selection map. For proportional, tournament, and ranking selection, $\frac{d \mathcal{F}}{d x}\left(e_{0}\right)$ is twist separative. The matrices $\frac{\partial \mathcal{H}}{\partial x}(v, 0)$ and $\left(\frac{\partial \mathcal{H}}{\partial x}(v, 0)\right)^{-1}$ are also twist separative (where $\mathcal{H}(x, p)=\mathcal{G}(x, p)-x)$.
Proof. Lemma 10 gives the formula for the differential. (Since we are taking the differential at $e_{0}$, the $\sigma_{0}$ permutation matrix is the identity.)

$M(0)^{*}$ is twist separative by lemma 23 . Let the fitness vector be $\left\langle f_{0}, f_{1}, \ldots, f_{n-1}\right\rangle^{T}$. For proportional and tournament selection, lemmas 1 and corollary 6 show that $\frac{d \mathcal{F}}{d x}\left(e_{0}\right)$ is upper triangular with nonzero entries only on the diagonal and in the upper row. Theorem 7.3 of [13] can be used to prove the same for ranking selection. Thus, $\frac{d \mathcal{F}}{d x}\left(e_{0}\right)$ is twist separative. $\frac{\partial \mathcal{H}}{\partial x}\left(e_{0}, 0\right)=\frac{\partial \mathcal{G}}{\partial x}\left(e_{0}, 0\right)-I$ is clearly twist separative, and lemma 25 shows that $\left(\frac{\partial \mathcal{H}}{\partial x}\left(e_{0}, 0\right)\right)^{-1}$ is twist separative.

The previous lemma depends on our rearrangement of coordinates so that $v=e_{0}$. Without this assumption, $\sigma_{s} \frac{d \mathcal{F}}{d x}\left(e_{0}\right) \sigma_{s}$ is twist separative.

LEMma 29. For proportional, ranking, and tournament selection, $\frac{\partial \mathcal{G}}{\partial x}(v, 0)=\frac{\partial \mathcal{G}}{\partial x}\left(e_{0}, 0\right)$ is upper triangular and its diagonal entries are its eigenvalues $\lambda_{0}, \lambda_{1}, \ldots, \lambda_{n-1}$. For proportional selection, $\lambda_{0}=0$ and for $j$ such that $\mathbf{1}^{T} j=1$, $\frac{\partial \mathcal{G}}{\partial x}(v, 0)_{0, j}=-\lambda_{j}$.

Proof. The proof of lemma 28 shows that $\frac{d \mathcal{F}}{d x}\left(e_{0}\right)$ is upper triangular with nonzero elements only on the diagonal and in the upper row. For proportional selection, equation 4 shows that $\mathbf{1}^{T} \frac{d \mathcal{F}}{d x}\left(e_{0}\right)=0$. Since $\frac{d \mathcal{F}}{d x}\left(e_{0}\right)$ is twist separative, the only nonzero entries in column $j$ where $\mathbf{1}^{T} j=1$ are the row 0 entry and the diagonal entry. Thus, the row 0 entry must be the negative of the diagonal entry $\lambda_{j}$.

Since $\frac{\partial \mathcal{G}}{\partial x}(v, 0)$ is upper triangular, its diagonal entries are its eigenvalues $\lambda_{0}=0, \lambda_{1}, \ldots, \lambda_{n-1}$.

Theorem 30. Assume proportional selection. Let $v$ be a hyperbolic fixed point of $\mathcal{G}_{0}$. Then

$$
\frac{d h}{d p}(0)_{i}=\left\{\begin{array}{ll}
-\sum_{j: 1_{j=1}} \frac{1}{1-\lambda_{j}} & \text { if } i=0 \\
\frac{1}{1-\lambda_{i}} & \text { if } \mathbf{1}^{T} i=1 \\
0 & \text { otherwise }
\end{array} .\right.
$$

where $\lambda_{i}$ is the $i^{\text {th }}$ diagonal entry and eigenvalue of $\frac{\partial \mathcal{G}}{\partial x}(v, 0)$.

Proof. Lemma 19 shows that

$$
\left.\frac{d h}{d p}(0)=-\left(\frac{\partial \mathcal{H}}{\partial x}(v, 0)\right)^{-1} \frac{\partial \mathcal{H}}{\partial p}(v, 0)\right) .
$$

Since the identity map does not depend on $p$, $\left.\frac{\partial \mathcal{H}}{\partial p}(v, 0)\right)=$ $\left.\frac{\partial \mathcal{G}}{\partial p}(v, 0)\right)$ which is given by equation $(20)$. This shows that $\frac{d h}{d p}(0)_{i}=0$ for $i$ such that $i \neq 0$ and $\mathbf{1}^{T} i \neq 1$.

Let $E$ denote the matrix $\left(\frac{\partial \mathcal{H}}{\partial x}(v, 0)\right)^{-1}$. Note that the $i^{\text {th }}$ diagonal entry of the upper triangular matrix $\frac{\partial \mathcal{H}}{\partial x}(v, 0)$ is $\lambda_{i}-1$. and therefore the $i^{\text {th }}$ diagonal entry of $E$ is $\frac{1}{1-\lambda_{i}}$.

Lemma 28 shows that $E$ is twist separative. Then

$$
\left.\frac{d h}{d p}(0)_{i}=\sum_{j} E_{i, j} \frac{\partial \mathcal{H}}{\partial p}(v, 0)\right)_{j} .
$$

First, we consider $i>0$ and the $j^{\text {th }}$ entry in this sum. By equation $\left.(20) \frac{\partial \mathcal{H}}{\partial p}(v, 0)\right)_{j}$ is nonzero only when $j=0$ or 
$\mathbf{1}^{T} j=1$, and in this latter case $\left.\frac{\partial \mathcal{H}}{\partial p}(v, 0)\right)_{j}=1$. Since $E$ is twist separative, $E_{i, j}$ is nonzero only when $i=i \otimes j$. Thus, the only $j$ for which the $j^{\text {th }}$ term in the summation of equation (21) is nonzero is when $i=j$. Thus, when $i>0$, $\frac{d h}{d p}(0)_{i}=E_{i, i}=\frac{1}{1-\lambda_{i}}$.

Now we consider the $j^{\text {th }}$ entry in the sum of equation (21) when $i=0$.

Since $\left.\frac{\partial \mathcal{H}}{\partial p}(v, 0)\right)_{j}=0$ except when $\mathbf{1}^{T} j=1$ and $j=0$, we only need to consider entries $E_{0, j}$ where $\mathbf{1}^{T} j=1$ and $j=0$.

Lemma 29 shows that $\frac{\partial \mathcal{G}}{\partial x}(v, 0)_{0,0}=0$. Thus, 29 shows that $\frac{\partial \mathcal{H}}{\partial x}(v, 0)_{0,0}=E_{0,0}=-1$.

From lemma 26 and lemma 29 it follows that if $\mathbf{1}^{T} j=1$, $E_{0, j}=\frac{\lambda_{j}}{-\left(\lambda_{j}-1\right)}=\frac{\lambda_{j}}{1-\lambda_{j}}$. Thus,

$$
\left(\frac{d h}{d p}\right)_{0}=\ell+\sum_{j: 1^{T} j=1} \frac{\lambda_{j}}{1-\lambda_{j}}=\sum_{j: \mathbf{1}^{T} j=1} \frac{1}{1-\lambda_{j}} .
$$

Let $B_{\varepsilon}(v)=\left\{x \in \mathbb{R}^{n}:\|x-v\|<\varepsilon\right.$ be the ball of radius $\epsilon$ around $v$. The next theorem shows that as mutation increases, a hyperbolic asymptotically stable fixed point moves into the interior of the simplex.

THEOREM 31. Assume proportional selection, and assume that $v$ is a hyperbolic asymptotically stable fixed point of $\mathcal{G}_{0}$, and $h$ is the map defined in lemma 19. There is an $\varepsilon>0$ and $a \gamma>0$ such that for $p \in[0, \gamma), h(p)$ is the unique fixed point of $\mathcal{G}_{p}$ in $B_{\epsilon}(v)$.

Proof. Lemma 22 shows that $\frac{\partial \mathcal{G}}{\partial p}(v, 0)=\frac{\partial \mathcal{H}}{\partial p}(v, 0) \neq 0$. Thus, $\frac{d h}{d p}(0) \neq 0$. By the continuity of the derivative, there is a $\eta>0$ such that for $0 \leq q \leq \eta, \frac{d h}{d p}(q)^{T} \frac{d h}{d p}(0)>0$. We can also assume that $\eta<\delta$ for the $\delta$ of lemma 20. Let $\gamma=\|v-h(q)\|$. Then for such $q,\|h(q)-v\|$ is strictly monotonically increasing as $q$ increases. Let $\varepsilon>0$ be such that $\varepsilon \leq\|h(\eta)-v\|$ and $B_{\varepsilon}(v)$ gives the unique fixed point of lemma 20. Now choose $\gamma>0$ to be sufficiently small that if $0<p<\gamma<\delta$, then $h(p) \in B_{\varepsilon}$. Then $h(p)$ must be the unique fixed point of lemma 20.

\subsection{Discussion}

Let $v$ be a hyperbolic vertex fixed point corresponding to a uniform population of $k$ individuals, i. e., $v=e_{k}$. Theorem 16 shows that for proportional selection each eigenvalue of $\frac{\partial \mathcal{G}}{\partial x}(v, 0)$ (other than 0 ) is determined by the fitness and the properties of some other point $i \oplus k$ of the search space. Theorem 30 shows that these fitnesses for the Hamming distance 1 neighbors of $k$ determine the direction of $\frac{d h}{d p}(0)$. If all Hamming distance 1 neighbors have fitness less than the fitness of $k$, then the vector from $v$ to $\frac{d h}{d p}(0)$ points in the direction of the interior of the face of $\Lambda$ determined by $v$ and its Hamming distance 1 neighbors. If some Hamming distance 1 neighbor has greater fitness, then the vector from $v$ to $\frac{d h}{d p}(0)$ points outside of the simplex (providing an alternate proof of theorem 21 in this special case).

If $v=e_{k}$ is asymptotically unstable under zero mutation, this means that at least one eigenvalue of $\frac{\partial \mathcal{G}}{\partial x}(v, 0)$ is greater than 1. Lemma 16 shows that each eigenvalue corresponds to some point in the search space other than $k$ which has higher fitness than $k$. The instability of $v$ means that when the SGA IPM is initialized a population which is both near to $v$, interior to the simplex, and not on the stable manifold of $v$, it will diverge away from $v$ due to the influence of the higher fitness points just mentioned..

Any population vector interior to the simplex must contain a nonzero representation of every point in the search space, including those higher fitness points that caused $v$ to be unstable. But in a finite GA population in a situation where the string length is realistically long, this won't happen since the size of the search space grows exponentially with the string length. If the search space points that make $v$ unstable have a large Hamming distance from $v$, they are unlikely to be included in a finite population near $v$. And if there is mutation with a mutation rate that is $\Theta(1 / n)$, such points are unlikely to be discovered in a realistic time period. Thus, from the point of view of finite populations, $v$ is stable in that when a with-mutation GA is at a population near $v$, it is likely to remain there for a long time (namely, until the higher-fitness points that make $v$ unstable are discovered by mutation). In the case of no-mutation, we would conjecture that the GA would be likely to be absorbed into the population corresponding to $v$.

We can also conjecture that when the search space points that make $v$ unstable at at least Hamming distance $j$ from $v$, then as the mutation rate $p$ increases from 0 , the fixed point corresponding to $v$ is order $O\left(p^{j}\right)$ close to the face of the simplex determined by $v$ and its Hamming distance 1 neighbors. In other words, it would be very close to the simplex. When a saddle-point fixed point is very close to but not in the simplex, the IPM will move very slowly when close to this fixed point $[13 ; 12]$.

\subsubsection{Examples}

We give two examples of the situation just described. Both use proportional selection, 1-point crossover with crossover rate $7 / 8$ and the following fitness function:

$$
f(i)= \begin{cases}20 & \text { if } \mathbf{1}^{T} i=0 \\ 165 & \text { if } \mathbf{1}^{T} i=\ell \\ 1 & \text { otherwise }\end{cases}
$$

For any string length and 1-point crossover with rate $7 / 8$, the uniform population consisting of the all zeros string is an unstable vertex fixed point because the eigenvalue corresponding to the all ones string is $\frac{33}{32}$ by theorem 16 .

In the first example the SGA IPM (a model) is started at the all zeros string, which has fitness 20 . The string length is 7 and the mutation rate is $\frac{1}{100}$. The all ones string, which has fitness 165 , has a frequency of $10^{-14}$ after one time step. This frequency gradually increases. After one time step the fitness is 18.71, and this decreases to 18.641164 after 6 time steps. For the next 145 time steps, the fitness is very gradually decreasing, but to 8 significant digits, it remains at 18.641164. After 369 time steps, the fitness reaches a minimum of 18.272 and then it increases rapidly reaching 153.71 at time step 376 . The IPM converges to within a tolerance of $10^{-12}$ after 382 time steps with a fitness of 153.79 .

In the second example, the SGA (an actual GA) is run with the same fitness, string length 9 , mutation rate $\frac{1}{10}$, and population size 1000 . Initialization is with multiple copies of the all zeros string. The SGA was run until it found the optimum. With 100 runs, the average number of generations to find the optimum was 37,645 with a standard deviation of 34,767 . The number of fitness evaluations was approximately 1000 times the number of generations. Clearly, while 
the all zeros uniform population is unstable as a fixed point, the GA takes a very long time to leave a neighborhood of this fixed point.

\section{FIXED POINT MOVEMENT EXAMPLES}

Next we will look in detail at gross movement of vertex fixed points for NEEDLE and BINEEDLE under varying mutation and crossover rates. The below is done via analysing and numerically iterating the $\mathcal{G}$-map, not with any running GA.

\subsection{Stability of BINEEDLE Fixed Points}

We assume that $\ell=2, a=1$, and no mutation. Interestingly, the center of the simplex $\langle 1 / 4,1 / 4,1 / 4,1 / 4\rangle$ is on the stable manifold of an unstable fixed point in the interior of the simplex. Thus, this fixed point can be found by iterating $\mathcal{G}$ starting at this point of the simplex.

The stability of the BINEEDLE fixed points can either be determined by directly computing the eigenvalues of the differential of $\mathcal{G}$ at the fixed point, or, in the case of vertex fixed points, by applying theorem 16 . However, when directly computing the eigenvalues, there will be one eigenvalue that corresponds to a direction outside of the hyperplane of the simplex, and this eigenvalue is not relevant to the stability of $\mathcal{G}$ in the simplex. Theorem 16 says that this eigenvalue is 0 for proportional selection and for vertex fixed points.

Table 1 contains the results of doing this for these five fixed points of the $\mathcal{G}$-map with one-point crossover rate $c=$ 1.0 and mutation rate $p=0$ applied to the BINEEDLE fitness function. The eigenvalues not in the direction of the hyperplane of the simplex are not included.

Table 2 contains the same results for uniform crossover with crossover rate 1.

Table 1: BINEEDLE vertex fixed points, one-point crossover rate 1 and no mutation.

\begin{tabular}{|l|c|r|} 
population & eigenvalues & type \\
\hline$\langle 1,0,0,0\rangle$ & {$[1 / 2,1 / 2,0]$} & Stable \\
$\langle 0,1,0,0\rangle$ & {$[2,2,0]$} & Saddle \\
$\langle 0,0,1,0\rangle$ & {$[2,2,0]$} & Saddle \\
$\langle 0,0,0,1\rangle$ & {$[1 / 2,1 / 2,0]$} & Stable \\
$\langle 1 / 4,1 / 4,1 / 4,1 / 4\rangle$ & {$[4 / 3,2 / 3,0]$} & Saddle
\end{tabular}

Table 2: BINEEDLE vertex fixed points, uniform crossover rate 1 and no mutation.

\begin{tabular}{|l|c|r|} 
population & eigenvalues & type \\
\hline$\langle 1,0,0,0\rangle$ & {$[1 / 2,1 / 2,1 / 2]$} & Stable \\
$\langle 0,1,0,0\rangle$ & {$[2,2,1 / 2]$} & Saddle \\
$\langle 0,0,1,0\rangle$ & {$[2,2,1 / 2]$} & Saddle \\
$\langle 0,0,0,1\rangle$ & {$[1 / 2,1 / 2,1 / 2]$} & Stable \\
$\langle 0.3202,0.1798$, & & \\
$0.1798,0.3202\rangle$ & {$[1.219,0.6906,0.3716]$} & Saddle
\end{tabular}

In both cases, simplex points of the form $\langle a, b, b, a\rangle$ with $a+b=1 / 2$ are on the stable manifold of the fixed point which is interior to the fixed point, but as soon as the symmetry of points in this form is broken, then such points are in the domain of attraction of one of the stable fixed points.

It is interesting to note that one-point crossover with rate 1 for $\ell=2$ takes any population to linkage equilibrium in one step. Thus, it is the same as gene pool crossover. This is not true for string lengths longer than 2 .

\subsection{Stability of points on the segment between vertices of maximum fitness}

Define a variant of the NEEDLE for $\ell=4, n=2^{4}=16$ as follows:

$$
\text { CONCATNEEDLE : }\langle 2,2,1, \ldots, 1\rangle
$$

For a population of $x=\langle\alpha, \beta, 0, \ldots, 0\rangle$ with $\alpha+\beta=1$, it can be verified that $\mathcal{G}(x)=x$. Thus, there is a line segment of fixed points. At these fixed points, the differential of $\mathcal{G}$ has one eigenvalue of 1 and all other eigenvalues are less than 1 . The eigenvector corresponding to the eigenvalue of 1 is in the direction of the line between the vertices corresponding to binary strings 0000 and 0001 , the two points of higher fitness. This suggests that all points on this line segment are stable (but not asymptotically stable) fixed points.

\subsection{Introducing Epsilon Mutation}

For the BINEEDLE a computational study was done to attempt to determine where the vertex fixed points moved to under increasing mutation. Iteration experiments of the G-map were done with initial populations set at either of the two stable vertex fixed point populations or the simplexcenter population. String length $\ell=4$ and one-point crossover were used with crossover rate 1 . A range of mutation rates were chosen. Note that the experiment was explicitly done for both stable vertex-populations at all-ones and all-zeros. Note that the fitness function is symmetric under changing zeros to ones and ones to zeros, and the same is true of the $\mathcal{G}$ function when applied to such a fitness function. This symmetry of the fitness function means that fixed points are invariant under this symmetry, and for any fixed point that is not mapped to itself by this symmetry, there is another fixed point with the same stability properties which is the image of the original fixed point under the symmetry.

Three initial populations were used, one at the all-zeros needle, one at a center-of-simplex population, and one the midpoint population between the all-zeros and all-ones population.

Figures 1 and 2 both display an interpolated version of the bifurcation diagram. At each mutation rate the $\mathcal{G}$-map was initialized and iterated to convergence with each of the three initial populations. The fitness value of each converged map was recorded. The figures contain mutation rates on the $x$-axis and fitness values (or projections) on the $y$-axis.

The upper curve of Figure 1 displays both stable fixed points as the $y$-axis represents fitness and these points have the same fitness by symmetry. The lower curve displays the unstable saddle point's average fitness. The critical point of bifurcation is the mutation rate of $1 / 7.65$

This bifurcation is an instance of the pitchfork bifurcation, where a stable fixed point bifurcates into two stable fixed points and an unstable fixed point. The two stable fixed points are symmetric under changing zeros to ones and ones to zeros. In order to demonstrate this, we looked 


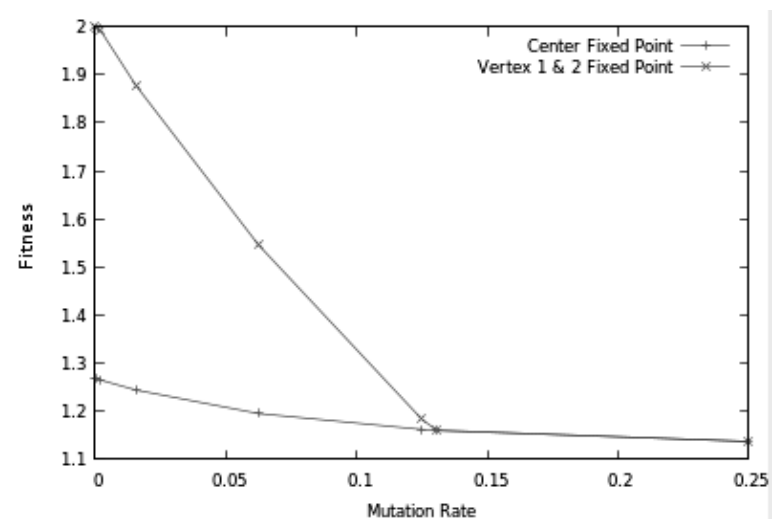

Figure 1: Epsilon mutation bifurcation of the stable fixed points. The $\mathrm{x}$-axis is the mutation rate $p$ and the $\mathbf{y}$-axis is fitness value of the f-p's population vector. The upper curve is actually two fixed-points with identical fitness values. At approximately $p=1 / 7.65$ the three distinct fixed points merge into one.

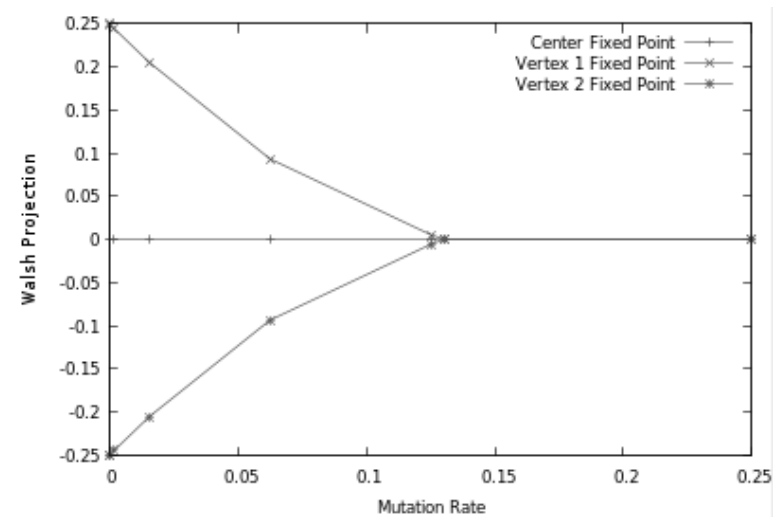

Figure 2: Epsilon mutation bifurcation of stable fixed points in the Walsh basis. The $\mathrm{x}$-axis is the mutation rate $p$ and the $\mathbf{y}$-axis is the Walsh projection. At approximately $p=1 / 7.65$ the three distinct fixed points merge into one.

for a projection of the fixed points that would demonstrate the pitchfork bifurcation. We found such a projection by representing the fixed points in the Walsh basis, which is described in [18].

Figure 2 shows the fixed points projected into coordinate 11 of their Walsh-basis representation as a function of the mutation rate. It shows a typical pitchfork bifurcation with a single stable fixed point splitting into two stable fixed points and an unstable fixed point which is "between" the stable fixed points.

As with the example with $\ell=2$, points that are invariant under the symmetry are on the stable manifold of the unstable fixed point, and thus the unstable fixed point can be found by iteration. For more general problems, it would be difficult to find points on the stable manifold of unstable fixed points, and so this procedure would be unlikely to work.

The above results are by definition incomplete, they might

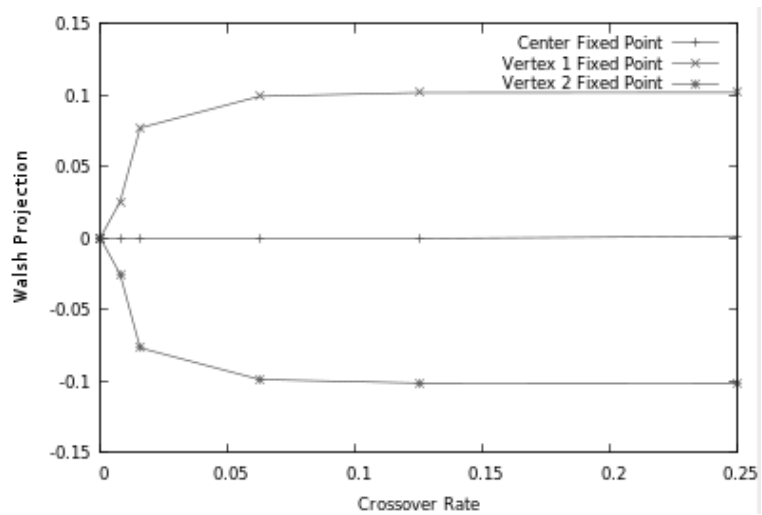

Figure 3: Epsilon crossover bifurcation of stable fixed points. The $\mathrm{x}$-axis is the crossover rate $c$ and the $y$-axis is the Walsh projection. At approximately $c=1 / 128$ the single fixed point splits into three.

not contain all fixed points. Many more initial populations would need to be tried, and unstable (non-saddle) fixed points are not observable via iteration. With small mutation rates, the overall dynamics have not changed much from mutation rate 0 . There still exist three observable fixed points, two stable and one saddle point. Yet it's clearly observable that at approximately mutation rate $1 / 7.65$ something interesting happens, a single stable fixed point splits into three fixed points.

\subsection{Introducing Epsilon Crossover}

A similar experiment was done examining the effects of adding epsilon one-point crossover to a fixed mutation rate G-map. The mutation rate is set to a mutation rate of $1 / n=$ $1 / 16$ (different than the classic $1 / \ell$ rate), and the crossover rate is varied over a range. Note that with zero crossover the Perron-Frobenius theorem implies that there exists only a single stable fixed point in the interior of the simplex. Figure 3 displays an interpolated version of the bifurcation diagram. As in the last subsection, the figure displays the projection into coordinate 11 of the Walsh basis representation.

These results are very interesting in that they indicate an observable bifurcation of a single stable fixed into a two stable fixed points and a saddle point. This happens at approximately crossover rate $1 / 128$. Over 8,000 iterations of the $\mathcal{G}$-map with this crossover rate were done starting from the uniform population consisting of the all-zeros strings. At this point that run was stopped, with the result seen in equation (23).

$$
\begin{array}{r}
\langle 0.3,0.05,0.049,0.016,0.049,0.016,0.016,0.032 \\
0.05,0.016,0.016,0.03,0.016,0.03,0.032,0.21\rangle
\end{array}
$$

The leading eigenvalue of the derivative at this stopping point was 0.99960 , meaning that the trajectory of iteration is very likely close to the stable manifold of the saddle point. The leading eigenvalue of the derivative at the fixed-point in row 4 was exactly 1 . Note that these numbers are approximate and at these types of critical points the numerics of computation in binary computers can result in some inaccuracies.

Once the crossover rate grew to $1 / 64$ and above a clear separation of iterative convergence was established between 
the different starting populations. At crossover rate 63/64 the interior saddle point's population approaches the center of the simplex population where all coordinates are equal to $1 / 16$.

\subsection{Revisiting Bistability}

We now give an example of the movement of fixed points when there is bistability.

The fitness function used for this example is the sloping needle SNEEDLE which is defined below. This is a special case of the sloping plateau fitness functions from [21].

$$
S N E E D L E_{a, b}(x)= \begin{cases}a+b+1 & \text { if } \mathbf{1}^{T} x=0 \\ b+\left(\ell-\mathbf{1}^{T} x\right) / \ell & \text { if } \mathbf{1}^{T} x>0\end{cases}
$$

Figure 4 shows a plot of SNEEDLE for $\ell=40, a=39$, $b=20$.

A key point of this fitness function is that the floor area of the function slopes directly to the needle. To a simple hillclimber $(1+1) E A$ this function is indistinguishable from a function like ZEROMAX (the inverse of ONEMAX). In addition it is easily solvable by an non-crossover EA/GA with an arbitrary population size. Just as with the sloping plateau, the function is designed to deceive proportional selection. In general, any EA with a large population will be slower to optimize this function than one with a small population where the effects of 'weak selection' are muted.

The infinite population model used for this example is a coarse graining of the SGA IPM to the equivalence classes defined by the equivalence $x \equiv y$ iff $\mathbf{1}^{T} x=\mathbf{1}^{T} y$. This unitation-based infinite population model (IPM) is described in more detail in [21]. When there is no crossover, the model accurately describes the behavior of the SGA IPM projected to unitation classes. However, with crossover, the model does not necessarily accurately described the behavior of the SGA IPM on the simplex when projected into unitation classes. But if we define the set $\Psi=\left\{x \in \Lambda: \mathbf{1}^{T} i=\right.$ $\mathbf{1}^{T} j$ implies $\left.x_{i}=x_{j}\right\}$ of populations in the simplex where all elements with the same unitation have equal representation, then the unitation-based IPM does accurately describe the behavior of the SGA IPM on $\Psi$, and that is what the example of this section shows. However, the unitation-based IPM may not accurately represent the stability of fixed points, and "stable" below means stable relative to the unitationbased IPM.

The string length is $\ell=40$. We hold the mutation rate fixed at $1 / 3 \ell$ and the $b$ parameter fixed at 20 , and vary the $a$ in a range in an effort to discover a bifurcation point. Uniform crossover with rate 1 was used. At each value of $a \in[1,40]$ the $\mathcal{G}$-map was iterated to convergence starting from two initial populations. The first is the center of the simplex population with equal weights on all individuals, and the second consists entirely of members on the needle at the all-zeros string.

For values of $a<21$ the IPM started from both initial populations converged to the same fixed point. At $a=21$ a bifurcation point is reached. The stable fixed point splits into the two stable fixed points shown in figure 5. In dynamical systems, this does not happen without there also being an unstable fixed point which is not shown. For increasing values of $a$, the average population fitness of the higher-fitness fixed point climbs until it reaches approximately average fitness of 41 and then levels off. The fixed point converged to

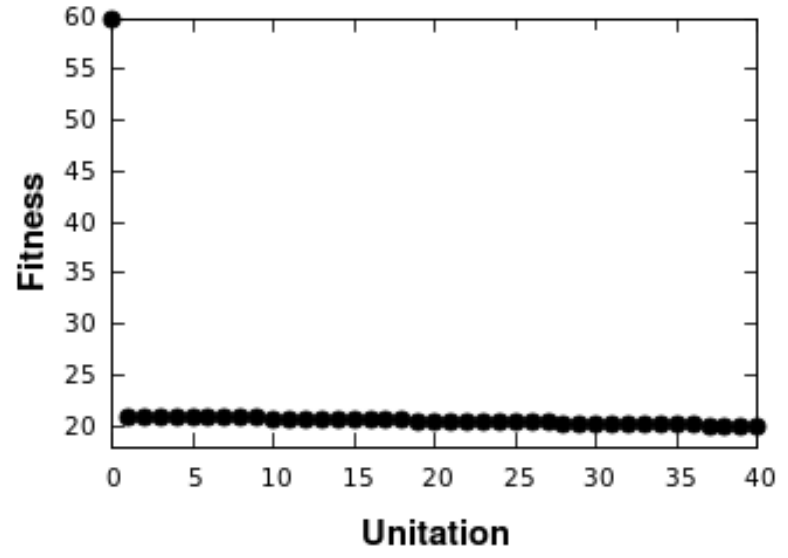

Figure 4: Sloping Needle Fitness, $\ell=40, k=1, a=$ $39, b=20$

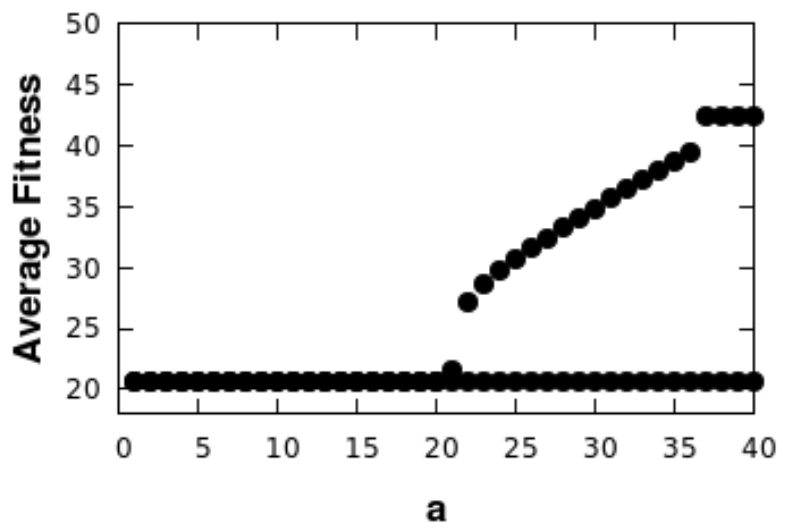

Figure 5: Sloping Needle fixed point bifurcation. The $\mathrm{x}$-axis is the $a$ parameter of SNEEDLE and the $y$-axis is the average fitness of the population vector. At approximately $a=21$ the fixed point splits into two.

from the initial center of the simplex population remains at avg-fitness $=20.5$ for all values of $a$ tested.

The resulting bifurcation diagram is pictured in Figure 5. The y-axis represents the weighted average fitness of a fixed point. As stated above, we cannot be sure that these "stable" fixed points are stable for the SGA IPM.

\section{CONCLUSIONS}

Populations consisting of multiple copies of a single bit string are vertex fixed points for the infinite population model (IPM) for the simple genetic algorithm (SGA) under zero mutation. For proportional selection, when these fixed points are hyperbolic (no eigenvalue of the differential has modulus 1), the stability of these fixed points is understood from previous work - see corollary 16. We have derived formulas for the stability of vertex fixed points under standard tournament selection with the assumption that no two individuals have the same fitness - see corollary 15 . However, the behavior of these fixed points as the mutation rate increases from zero was not known. We have shown that if a vertex fixed point is hyperbolic and asymptotically 
stable, then the fixed point moves into the interior of the simplex as the mutation rate increases from zero. If the vertex fixed point is hyperbolic and asymptotically unstable, then the simplex moves out of the simplex as the mutation rate increases from zero.

We have given examples of the behavior and bifurcation of fixed points as the mutation rate varies from 0 to $1 / 2$, and as the crossover rate varies when the mutation rate is held fixed.

In evolutionary computation, bistability is defined as two stable fixed points corresponding to one fitness peak. We have given an example of bistability for the sloping needle fitness function. This example shows how a stable fixed point bifurcates into two stable fixed points and an unstable fixed point as the height of the fitness function needle is increased. The example uses a unitation-based IPM which correctly describes the behavior of the SGA IPM on the subset of the simplex where populations have equal representations for all points in a unitation class.

Clearly, a direction for future work would be to rigorously describe the types of movement and bifurcation of fixed points under the variation of the parameters of mixing, especially the mutation and crossover rates. To make this task more tractable, there are two possible simplifying assumptions. The first is to use gene pool crossover as was done in [23], [22], and [20]. The second is to assume a fitness function of unitation (where the fitness of a binary string depends only on the number of ones in the string), and then to use the unitation-based IPM described above and in section 5.5. With these two assumptions, it may be possible to reduce the fixed point equations to equations in a single variable as was done in [23].

\section{ACKNOWLEDGMENTS}

T. G. was partially supported by NSF grant DMS-0818785, NSF CMMI grant 0849433 and DARPA grant 00001741/HR001109-1-0055.

\section{References}

[1] M. C. Boerlijst, S. Bonhoeffer, and M. A. Nowak. Viral quasi-species and recombination. Proc. Royal Society London B, 263:1577-1584, 1996.

[2] M. Brin and G. Stuck. Introduction to Dynamical Systems. Cambridge University Press, Cambridge, 2002.

[3] T. Gedeon, C. Hayes, and R. Swanson. Genericity of the fixed point set for the infinite population genetic algorithm. In Foundations of Genetic Algorithms (FOGA) 9, pages 97-109, 2007.

[4] D. Goldberg and K. Deb. A comparative analysis of selection schemes used in genetic algorithms. In Foundations of genetic algorithms 1, volume 1, pages 69-93. Morgan Kaufmann, 1991.

[5] C. Hayes and T. Gedeon. Hyperbolicity of the fixed point set for the simple genetic algorithm. Theoretical Computer Science, 411(25):2368 - 2383, 2010.

[6] C. Reeves and J. Rowe. Genetic algorithms: principles and perspectives: a guide to GA theory. Kluwer Academic Pub, 2002.
[7] J. N. Richter, J. Paxton, and A. H. Wright. EA models and population fixed-points versus mutation rates for functions of unitation. In H.-G. Beyer and U.M. O'Reilly, editors, GECCO, pages 1233-1240. ACM, 2005.

[8] J. N. Richter, A. Wright, and J. Paxton. Exploration of population fixed points versus mutation rates for functions of unitation. In K. D. et al., editor, GECCO, volume 3102 of Lecture Notes in Computer Science. Springer, 2004.

[9] C. Robinson. Dynamical Systems: Stability, Symbolic Dynamics, and Chaos. The CRC Press, 2nd edition, 199.

[10] J. E. Rowe. Population fixed-points for functions of unitation. In W. Banzhaf and C. R. Reeves, editors, FOGA, pages 69-84. Morgan Kaufmann, 1998.

[11] H. Suzuki and Y. Iwasa. Crossover accelerates evolution in gas with a babel-like fitness landscape: Mathematical analyses. Evolutionary Computation, 7(3):275-310, 1999.

[12] E. van Nimwegen. The Statistical Dynamics of Epochal Evolution. PhD thesis, Utrecht University, Utrecht, The Netherlands, 1999.

[13] M. D. Vose. The Simple Genetic Algorithm: Foundations and Theory. MIT Press, Cambridge, MA, 1999.

[14] M. D. Vose and G. E. Liepins. Representational issues in genetic optimization. Journal of Experimental and Theoretical Artificial Intelligence, 2:101-115, 1989.

[15] M. D. Vose and A. H. Wright. Simple genetic algorithms with linear fitness. Evolutionary Computation, 4(2):347-368, 1994.

[16] M. D. Vose and A. H. Wright. Stability of vertex fixed points and applications. In L. D. Whitley and M. D. Vose, editors, Foundations of genetic algorithms 3, pages 103-113, San Mateo, 1995. Morgan Kaufmann.

[17] M. D. Vose and A. H. Wright. The Walsh transform and the theory of the simple genetic algorithm. In S. Pal and P. Wang, editors, Genetic Algorithms for Pattern Recognition, pages 25-44. CRC Press, 1996.

[18] M. D. Vose and A. H. Wright. The simple genetic algorithm and the Walsh transform: Part I, theory. Evolutionary Computation, 6(3):253-273, 1998.

[19] A. H. Wright and G. L. Bidwell. A search for counterexamples to two conjectures on the simple genetic algorithm. In R. K. Belew and M. D. Vose, editors, Foundations of Genetic Algorithms (FOGA-4), pages 73-84. Morgan Kaufmann, 1997.

[20] A. H. Wright and G. Cripe. Bistability of the needle function in the presence of truncation selection. In GECCO 2004: Proceedings of the Genetic and Evolutionary Computation Conference. Springer Verlag, 2004.

[21] A. H. Wright and J. N. Richter. Strong recombination, weak selection, and mutation. In M. Cattolico, editor, GECCO, pages 1369-1376. ACM, 2006. 
[22] A. H. Wright, J. E. Rowe, R. Poli, and C. R. Stephens. A fixed point analysis of a gene pool GA with mutation. In Proceedings of the Genetic and Evolutionary Computation Conference (GECCO), San Francisco, CA., 2002. Morgan Kaufmann Publishers.

[23] A. H. Wright, J. E. Rowe, R. Poli, and C. R. Stephens. Bistability in a gene pool GA with mutation. In J. E. Rowe, K. DeJong, and R. Poli, editors, Foundations of Genetic Algorithms (FOGA-7), San Mateo, 2003. Morgan Kaufmann. 